Dose-Response Modeling Under Simple Order Restrictions Using Bayesian Variable Selection Methods

Supplementary material

OTAVA, Martin; SHKEDY, Ziv; Lin, Dan; GOEHLMANN, Hinrich W.H.; BIJNENS, Luc; TALLOEN, Willem \& KASIM, Adetayo (2014) Dose-Response Modeling Under Simple Order Restrictions Using Bayesian Variable Selection Methods. In: Statistics in Biopharmaceutical Research, 6 (3), p. 252-262.

DOI: $10.1080 / 19466315.2013 .855472$

Handle: http://hdl.handle.net/1942/17144 


\section{Dose-Response Modeling Under Simple Order Restrictions Using Bayesian Variable Selection Methods Supplementary appendix}

Martin Otava et al. 


\section{INTRODUCTION}

This supplementary appendix contains additional materials that were not presented in the manuscript and provides detailed information about various topics mentioned in the article. In Section 2 we present the complete results of the simulation study discussed in Section 5 in the manuscript. In Section 3 we present materials related to the likelihood ratio test (LRT) while Section 4 is devoted to the multiple contrast tests (MCT). Design matrices related to the BVS model are presented in Section 5 and an additional case study, analysis of dose-response microarray experiment, is presented in Section 6.

\section{SIMULATION RESULTS}

The simulation settings correspond to the setting used by Marcus (1976), except for the ordering of the models and more $\lambda$ values. The values for all profiles of $K=5$ (Table 11) were derived in the same way as for $K=4$. The setting matrices (Table 12 and Table 13) were multiplied by $\lambda$ to cover diverse relative differences among the dose levels. Using $\lambda=0$ shows behaviour under the null model, for the alternative models we set $\lambda=1,1.5,2,2.5,3$. Afterwards, for each setting, 1000 data were generated. Each dataset contains four dose levels (control included) with varying number of samples $(n=3,4,5)$ per dose. The noise followed a standard normal distribution. All the methods were applied under the assumption of the monotone upward trend for investigating the power and under the null model for investigating the Type I error. One-sided tests were run for the MCTs and the LRT. For $K=4$ and $n=4$, simulation was repeated with different choices of variance of Gaussian distribution (Figure 5).

Figure 1, 2, 3 and 4 shows comparison of p-values of the frequentist methods and the posterior probabilities of the null model of the BVS for $K=4$. Both quantities are very different, so we do not expect their correspondence (i.e., points around plotted diagonal line). Still, the figures provides a nice visualization of the facts appearing in Table 1 and Tables 2 and 3 as well. The BVS posterior probabilities are higher in absolute values comparing the p-values, hence to achieve similar results (in terms of the power, the Type I error or the number of significant genes identified) the higher threshold than 0.05 has to be used for the BVS. Note that with an increasing $n$, the overall power increases and the difference among the methods diminishes in a similar way as with the increasing $\lambda$. The behavior of Type I error is shown in Figure 5. Visualization of the results is shown in Figure 6 for all $n=3,4,5$. The corresponding results for $K=5$ are provided in Tables 4, 5, 6, 7, 8 and 9 . 

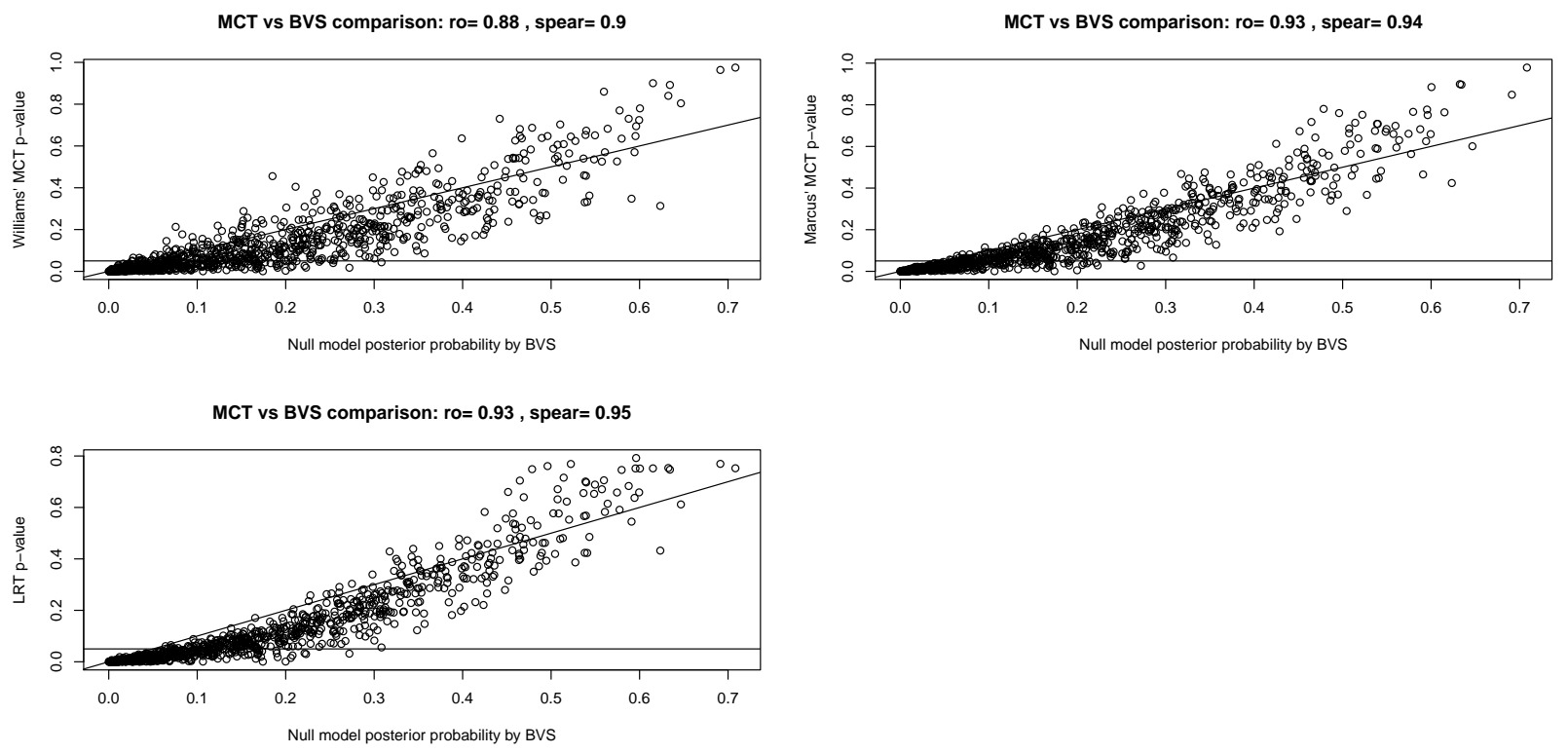

Figure 1: The p-values for the LRT and the MCTs against the posterior probabilities of the null hypothesis. Example for $K=4, \lambda=1$ and $g_{7}$. Top left: LRT vs. BVS. Top right: $M C T$ Williams vs. BVS, Bottom left: MCT Marcus vs. BVS.
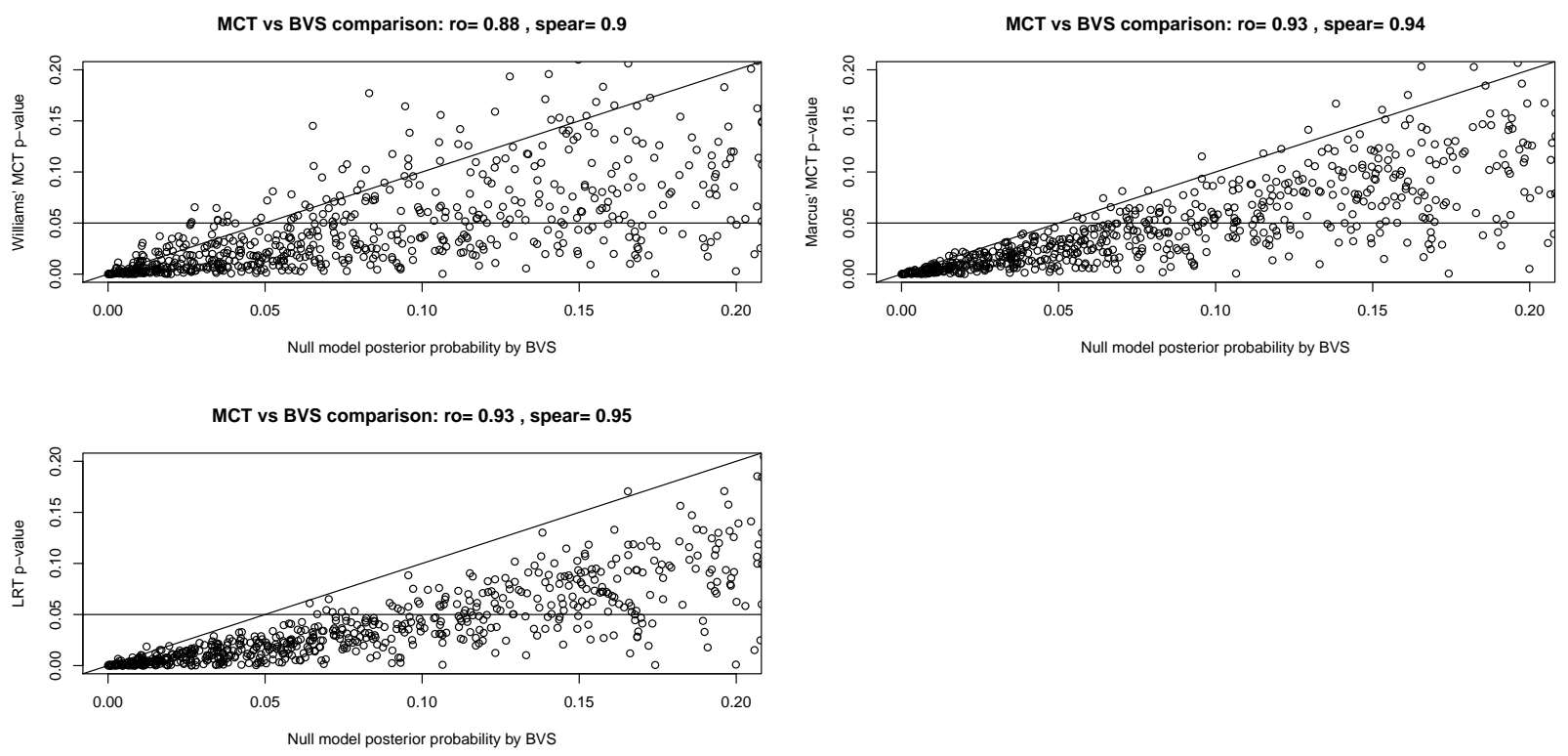

Figure 2: The p-values for the LRT and the MCTs against the posterior probabilities of the null hypothesis. Detail around the zero. Example for $K=4, \lambda=1$ and $g_{7}$. Top left: LRT vs. BVS. Top right: MCT Williams vs. BVS, Bottom left: MCT Marcus vs. BVS. 

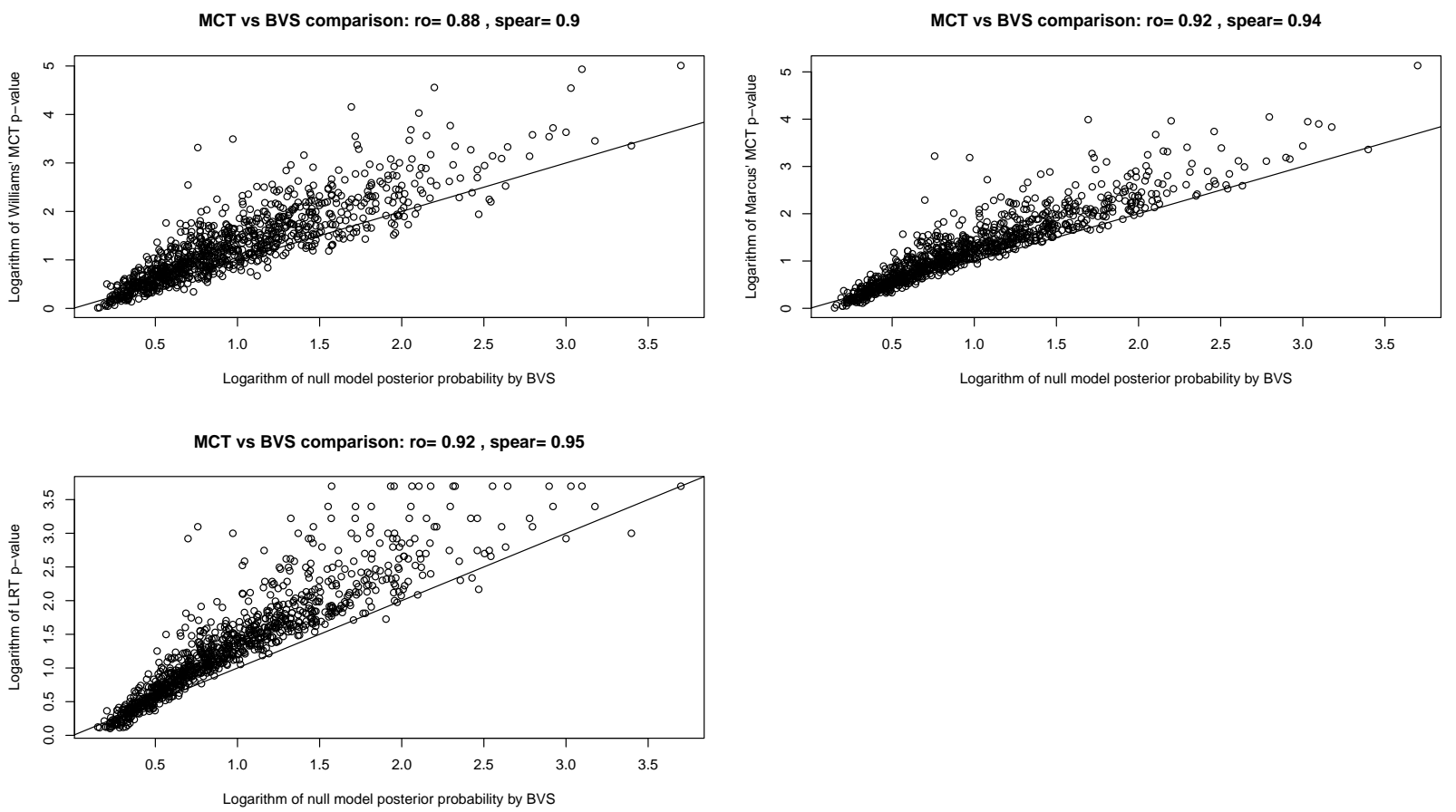

Figure 3: Logarithm of the p-values for the LRT and the MCTs against the logarithm of the posterior probabilities of the null hypothesis. Example for $K=4, \lambda=1$ and $g_{7}$. Top left: LRT vs. BVS. Top right: MCT Williams vs. BVS, Bottom left: MCT Marcus vs. BVS.
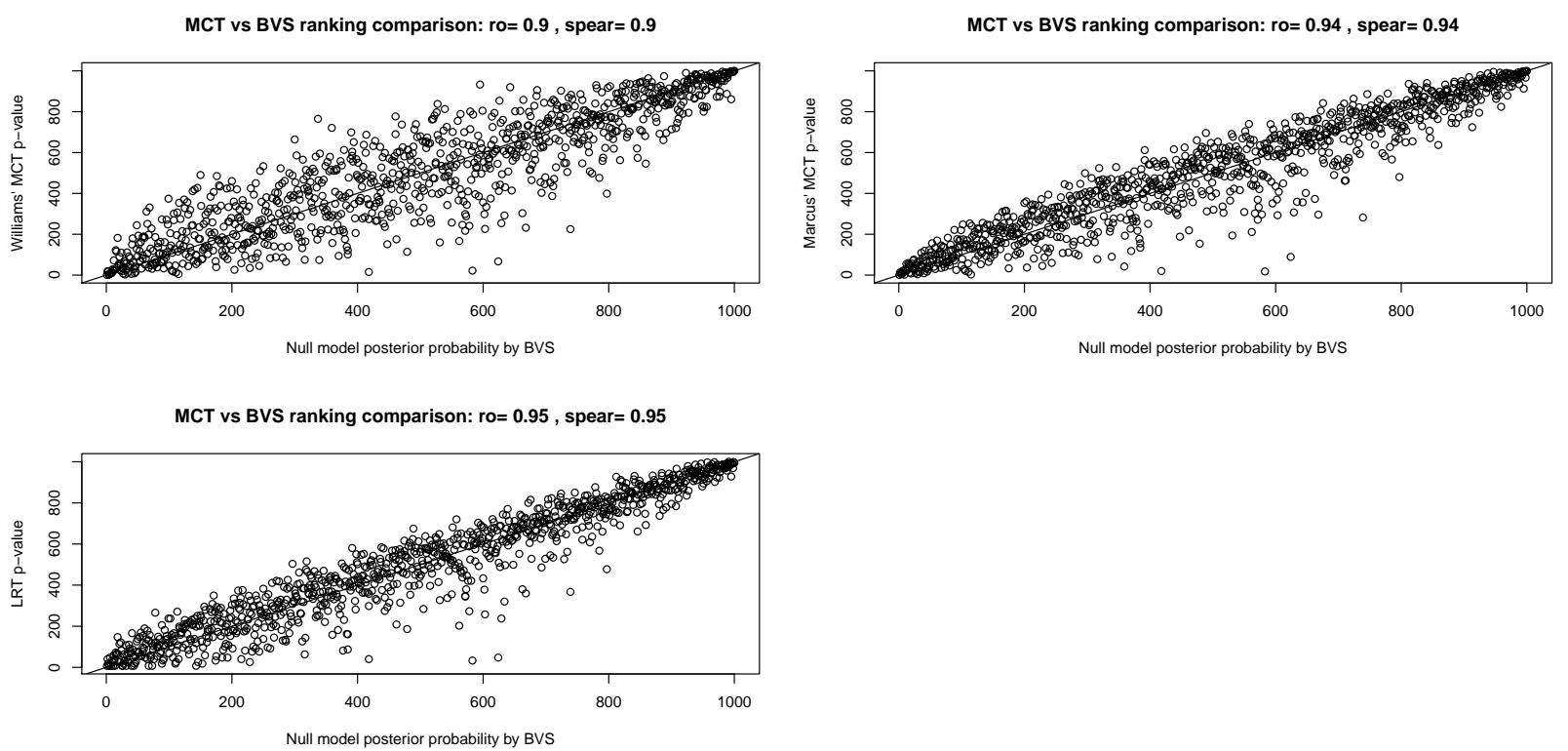

Figure 4: Ranking of the p-values for the LRT and the MCTs against the ranking of the posterior probabilities of the null hypothesis. Example for $K=4, \lambda=1$ and $g_{7}$. Top left: LRT vs. BVS. Top right: MCT Williams vs. BVS, Bottom left: MCT Marcus vs. BVS. 


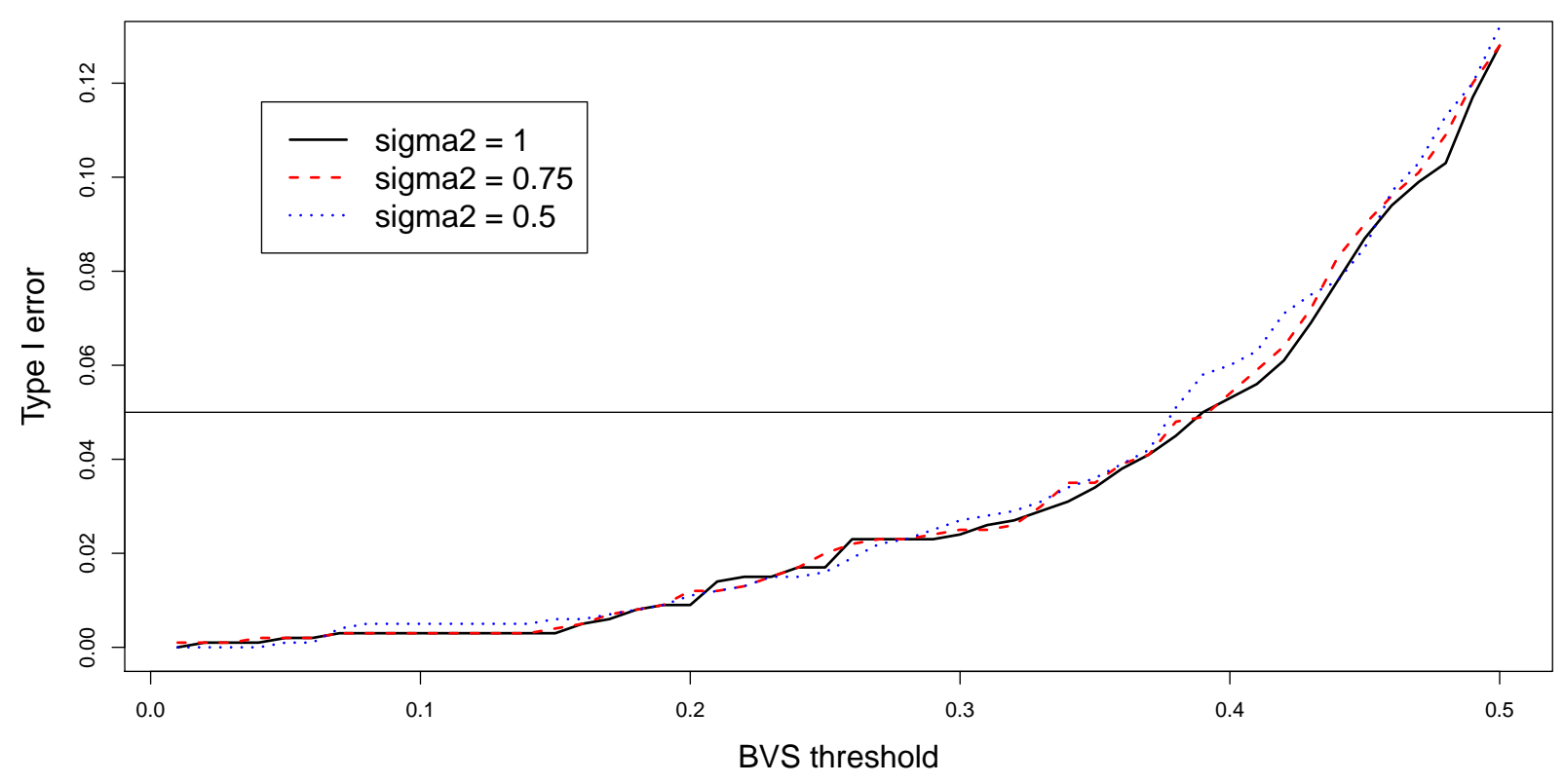

Figure 5: Proportion of the false rejections (Type I error) for the BVS when varying the threshold of a rejection of the null model. Based on 1000 simulated datasets with $n=4$ observations per dose with three values of $\sigma^{2}: \sigma^{2}=1$ (solid line), $\sigma^{2}=0.75$ (dashed line) and $\sigma^{2}=0.50$ (dotted line).
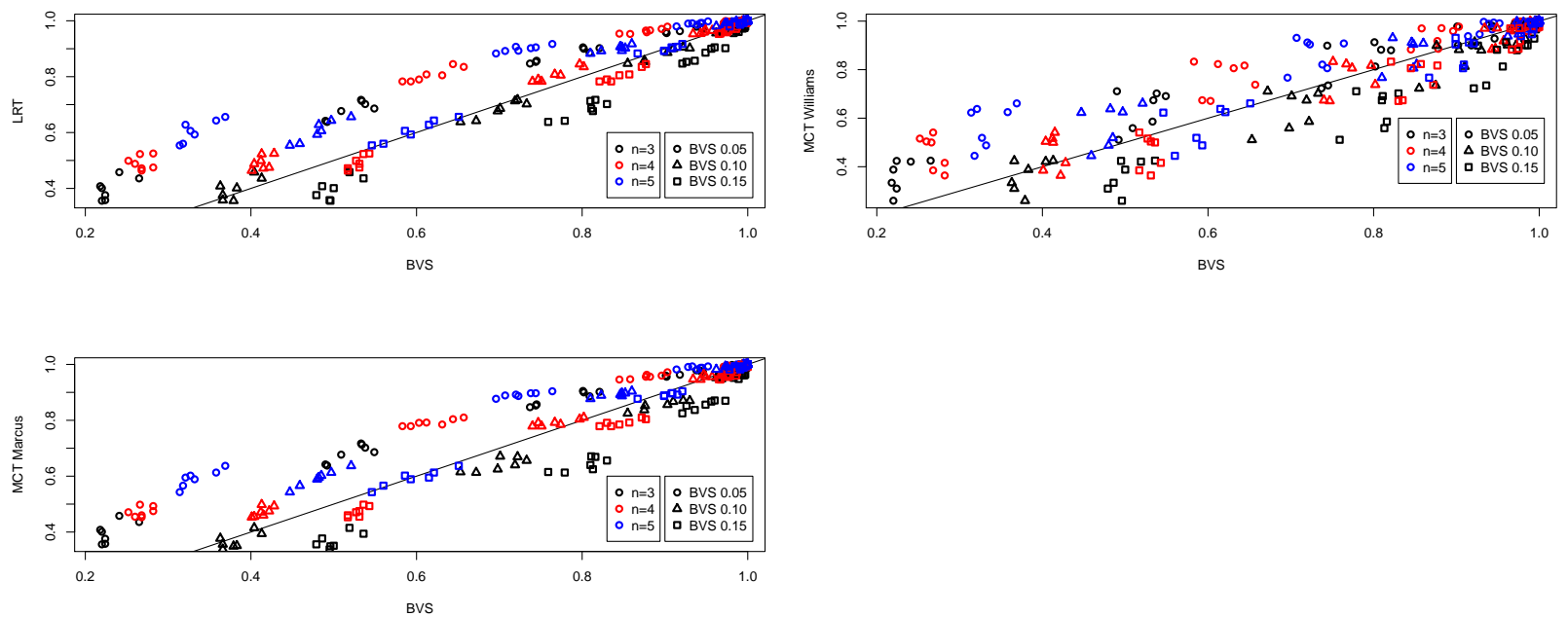

Figure 6: Comparison of the power between the BVS (with varying threshold) and the frequentist tests for $K=4$. Circles represent the results for the threshold $\alpha=0.05$, triangles $\alpha=0.10$ and rectangles $\alpha=0.15$. Black colour is related to the setting of $n=3$, red of $n=4$ and blue of $n=5$. Top left: LRT vs. BVS. Top right: MCT Williams vs. BVS, Bottom left: $M C T$ Marcus vs. BVS. 


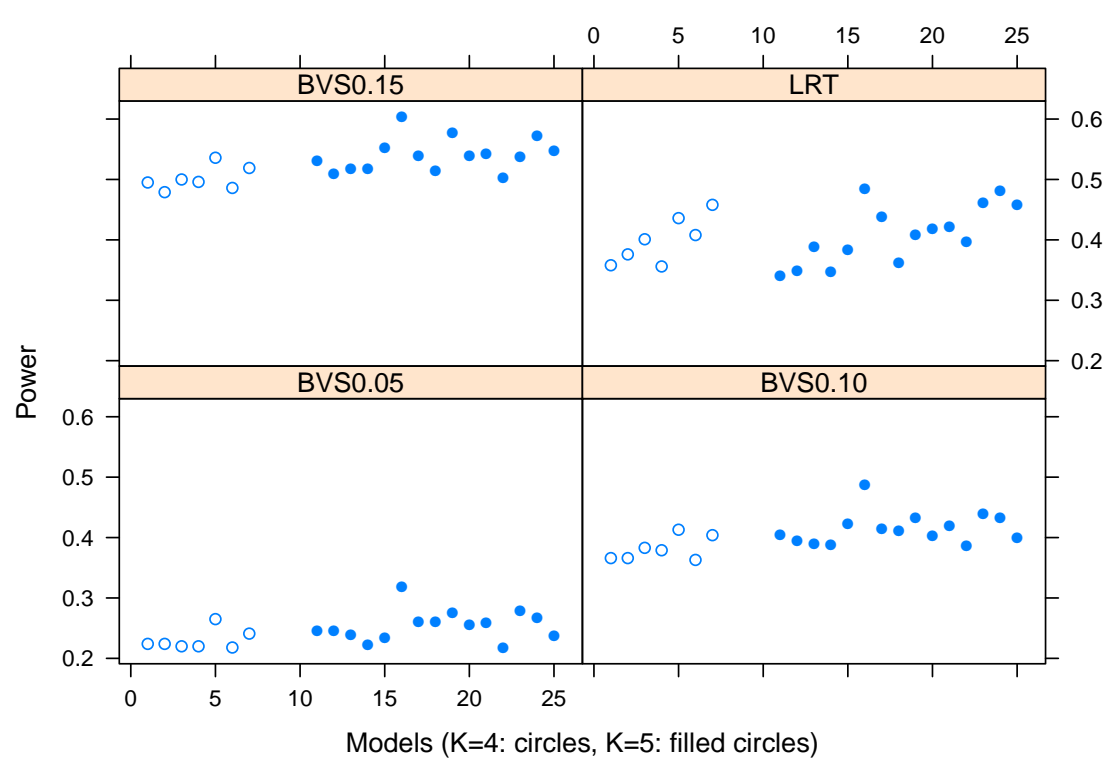

Figure 7: Comparison of the power between $K=4$ and $K=5$ for $B V S$ (with varying threshold) and LRT test (top right panel). The plot is based on a simulation under $\lambda=1$ and $n=3$. The models are ordered arbitrarily, seven models for $K=4$ on the left (circles) and 15 models for $K=5$ on the right (filled circles). 


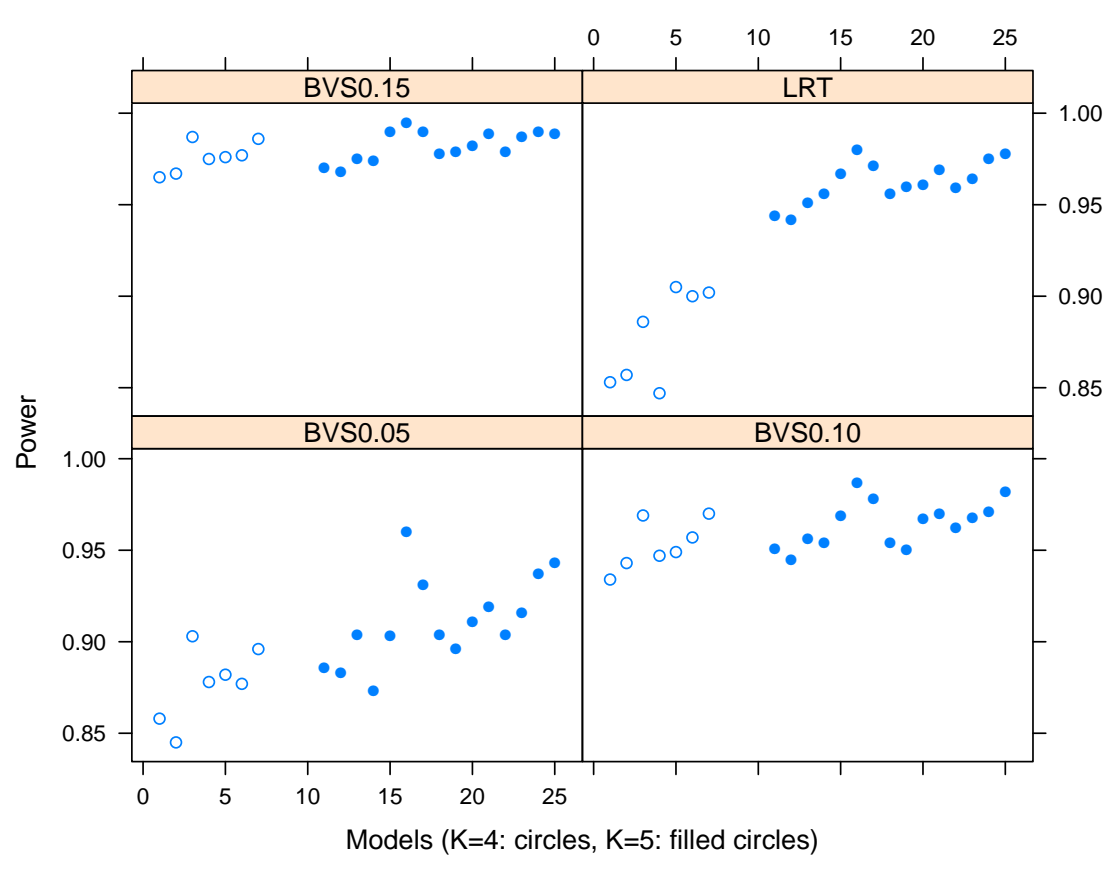

Figure 8: Comparison of the power between $K=4$ and $K=5$ for $B V S$ (with varying threshold) and LRT test (top right panel). The plot is based on a simulation under $\lambda=2$ and $n=4$. The models are ordered arbitrarily, seven models for $K=4$ on the left (circles) and 15 models for $K=5$ on the right (filled circles). 
Table 1: Results for $n=3$. Columns LRT and MCTs show estimation of the power of the particular tests. Columns BVS shows proportion of the (mean of) posterior probabilities of the null model given the data that are smaller then $\alpha=0.05,0.10,0.15,0.35$. Last column represent the estimated probability of the correct model having the highest mean of the posterior probability among all the possible models.

\begin{tabular}{|c|c|c|c|c|c|c|c|c|c|}
\hline$\lambda$ & Profile & LRT & $\begin{array}{r}\mathrm{MCT} \\
(\mathrm{W})\end{array}$ & $\begin{array}{r}\mathrm{MCT} \\
(\mathrm{M})\end{array}$ & $\begin{array}{l}\text { BVS } \\
0.05\end{array}$ & $\begin{array}{r}\text { BVS } \\
0.10\end{array}$ & $\begin{array}{r}\text { BVS } \\
0.15\end{array}$ & $\begin{array}{r}\text { BVS } \\
0.35\end{array}$ & True $\mathrm{m}$. \\
\hline \multirow[t]{7}{*}{1} & $g_{1}$ & 0.36 & 0.42 & 0.34 & 0.22 & 0.37 & 0.49 & 0.81 & 0.55 \\
\hline & $g_{2}$ & 0.38 & 0.31 & 0.36 & 0.22 & 0.37 & 0.48 & 0.80 & 0.46 \\
\hline & $g_{3}$ & 0.40 & 0.39 & 0.35 & 0.22 & 0.38 & 0.50 & 0.83 & 0.01 \\
\hline & $g_{4}$ & 0.36 & 0.26 & 0.35 & 0.22 & 0.38 & 0.50 & 0.83 & 0.59 \\
\hline & $g_{5}$ & 0.44 & 0.42 & 0.39 & 0.26 & 0.41 & 0.54 & 0.86 & 0.07 \\
\hline & $g_{6}$ & 0.41 & 0.33 & 0.38 & 0.22 & 0.36 & 0.49 & 0.82 & 0.02 \\
\hline & $g_{7}$ & 0.46 & 0.42 & 0.41 & 0.24 & 0.40 & 0.52 & 0.85 & 0.00 \\
\hline \multirow[t]{7}{*}{1.5} & $g_{1}$ & 0.64 & 0.71 & 0.61 & 0.49 & 0.67 & 0.78 & 0.95 & 0.73 \\
\hline & $g_{2}$ & 0.68 & 0.56 & 0.62 & 0.51 & 0.70 & 0.81 & 0.96 & 0.68 \\
\hline & $g_{3}$ & 0.69 & 0.69 & 0.67 & 0.55 & 0.70 & 0.81 & 0.96 & 0.08 \\
\hline & $g_{4}$ & 0.64 & 0.51 & 0.61 & 0.49 & 0.65 & 0.76 & 0.95 & 0.75 \\
\hline & $g_{5}$ & 0.70 & 0.70 & 0.66 & 0.54 & 0.73 & 0.83 & 0.97 & 0.21 \\
\hline & $g_{6}$ & 0.72 & 0.59 & 0.67 & 0.53 & 0.72 & 0.82 & 0.97 & 0.09 \\
\hline & $g_{7}$ & 0.71 & 0.67 & 0.64 & 0.53 & 0.72 & 0.81 & 0.97 & 0.00 \\
\hline \multirow[t]{7}{*}{2} & $g_{1}$ & 0.85 & 0.90 & 0.85 & 0.74 & 0.88 & 0.93 & 0.99 & 0.84 \\
\hline & $g_{2}$ & 0.86 & 0.73 & 0.84 & 0.74 & 0.88 & 0.94 & 0.99 & 0.78 \\
\hline & $g_{3}$ & 0.89 & 0.88 & 0.86 & 0.81 & 0.90 & 0.95 & 0.99 & 0.22 \\
\hline & $g_{4}$ & 0.85 & 0.72 & 0.82 & 0.74 & 0.85 & 0.92 & 0.99 & 0.84 \\
\hline & $g_{5}$ & 0.90 & 0.91 & 0.87 & 0.80 & 0.92 & 0.96 & 1.00 & 0.42 \\
\hline & $g_{6}$ & 0.90 & 0.81 & 0.87 & 0.80 & 0.91 & 0.96 & 1.00 & 0.23 \\
\hline & $g_{7}$ & 0.90 & 0.88 & 0.87 & 0.82 & 0.93 & 0.97 & 1.00 & 0.01 \\
\hline \multirow[t]{7}{*}{2.5} & $g_{1}$ & 0.96 & 0.98 & 0.95 & 0.90 & 0.97 & 0.99 & 1.00 & 0.86 \\
\hline & $g_{2}$ & 0.96 & 0.90 & 0.95 & 0.90 & 0.96 & 0.98 & 1.00 & 0.80 \\
\hline & $g_{3}$ & 0.98 & 0.98 & 0.96 & 0.94 & 0.98 & 1.00 & 1.00 & 0.39 \\
\hline & $g_{4}$ & 0.96 & 0.90 & 0.96 & 0.92 & 0.97 & 0.99 & 1.00 & 0.87 \\
\hline & $g_{5}$ & 0.98 & 0.98 & 0.96 & 0.94 & 0.98 & 0.99 & 1.00 & 0.63 \\
\hline & $g_{6}$ & 0.97 & 0.93 & 0.96 & 0.95 & 0.98 & 0.99 & 1.00 & 0.39 \\
\hline & $g_{7}$ & 0.97 & 0.98 & 0.96 & 0.94 & 0.98 & 1.00 & 1.00 & 0.04 \\
\hline \multirow[t]{7}{*}{3} & $g_{1}$ & 0.99 & 1.00 & 0.99 & 0.98 & 0.99 & 1.00 & 1.00 & 0.89 \\
\hline & $g_{2}$ & 0.99 & 0.98 & 0.99 & 0.99 & 1.00 & 1.00 & 1.00 & 0.84 \\
\hline & $g_{3}$ & 0.99 & 0.99 & 0.99 & 0.98 & 0.99 & 1.00 & 1.00 & 0.57 \\
\hline & $g_{4}$ & 0.99 & 0.97 & 0.99 & 0.97 & 0.99 & 1.00 & 1.00 & 0.89 \\
\hline & $g_{5}$ & 1.00 & 1.00 & 0.99 & 0.98 & 1.00 & 1.00 & 1.00 & 0.79 \\
\hline & $g_{6}$ & 1.00 & 0.98 & 0.99 & 0.99 & 1.00 & 1.00 & 1.00 & 0.58 \\
\hline & $g_{7}$ & 1.00 & 0.99 & 0.99 & 0.99 & 1.00 & 1.00 & 1.00 & 0.11 \\
\hline
\end{tabular}


Table 2: Results for $n=4$. Columns LRT and MCTs show estimation of the power of the particular tests. Columns BVS shows proportion of the (mean of) posterior probabilities of the null model given the data that are smaller then $\alpha=0.05,0.10,0.15$. Last column represent the estimated probability of the correct model having the highest mean of the posterior probability among all the possible models.

\begin{tabular}{|c|c|c|c|c|c|c|c|c|}
\hline$\lambda$ & Profile & LRT & $\begin{array}{r}\mathrm{MCT} \\
(\mathrm{W})\end{array}$ & $\begin{array}{r}\text { MCT } \\
(\mathrm{M})\end{array}$ & $\begin{array}{l}\text { BVS } \\
0.05\end{array}$ & $\begin{array}{c}\text { BVS } \\
0.10\end{array}$ & $\begin{array}{r}\text { BVS } \\
0.15\end{array}$ & True m. \\
\hline \multirow[t]{7}{*}{1} & $g_{1}$ & 0.47 & 0.54 & 0.46 & 0.27 & 0.41 & 0.52 & 0.58 \\
\hline & $g_{2}$ & 0.46 & 0.38 & 0.45 & 0.27 & 0.40 & 0.52 & 0.50 \\
\hline & $g_{3}$ & 0.50 & 0.52 & 0.47 & 0.25 & 0.41 & 0.53 & 0.01 \\
\hline & $g_{4}$ & 0.47 & 0.36 & 0.47 & 0.28 & 0.42 & 0.53 & 0.61 \\
\hline & $g_{5}$ & 0.49 & 0.50 & 0.46 & 0.26 & 0.40 & 0.53 & 0.06 \\
\hline & $g_{6}$ & 0.52 & 0.42 & 0.49 & 0.28 & 0.43 & 0.54 & 0.02 \\
\hline & $g_{7}$ & 0.52 & 0.50 & 0.50 & 0.27 & 0.41 & 0.54 & 0.00 \\
\hline \multirow[t]{7}{*}{1.5} & $g_{1}$ & 0.78 & 0.83 & 0.78 & 0.58 & 0.75 & 0.82 & 0.82 \\
\hline & $g_{2}$ & 0.78 & 0.67 & 0.78 & 0.59 & 0.74 & 0.83 & 0.75 \\
\hline & $g_{3}$ & 0.80 & 0.81 & 0.78 & 0.63 & 0.77 & 0.84 & 0.10 \\
\hline & $g_{4}$ & 0.79 & 0.67 & 0.79 & 0.60 & 0.75 & 0.83 & 0.81 \\
\hline & $g_{5}$ & 0.81 & 0.82 & 0.79 & 0.61 & 0.77 & 0.86 & 0.26 \\
\hline & $g_{6}$ & 0.83 & 0.74 & 0.81 & 0.66 & 0.80 & 0.87 & 0.12 \\
\hline & $g_{7}$ & 0.84 & 0.82 & 0.80 & 0.64 & 0.80 & 0.88 & 0.00 \\
\hline \multirow[t]{7}{*}{2} & $g_{1}$ & 0.95 & 0.97 & 0.95 & 0.86 & 0.93 & 0.96 & 0.86 \\
\hline & $g_{2}$ & 0.95 & 0.88 & 0.95 & 0.84 & 0.94 & 0.97 & 0.84 \\
\hline & $g_{3}$ & 0.98 & 0.98 & 0.97 & 0.90 & 0.97 & 0.99 & 0.29 \\
\hline & $g_{4}$ & 0.96 & 0.89 & 0.96 & 0.88 & 0.95 & 0.97 & 0.86 \\
\hline & $g_{5}$ & 0.97 & 0.97 & 0.96 & 0.88 & 0.95 & 0.98 & 0.52 \\
\hline & $g_{6}$ & 0.96 & 0.92 & 0.96 & 0.88 & 0.96 & 0.98 & 0.27 \\
\hline & $g_{7}$ & 0.97 & 0.96 & 0.96 & 0.90 & 0.97 & 0.99 & 0.02 \\
\hline \multirow[t]{7}{*}{2.5} & $g_{1}$ & 0.99 & 1.00 & 0.99 & 0.98 & 0.99 & 1.00 & 0.91 \\
\hline & $g_{2}$ & 1.00 & 0.98 & 1.00 & 0.97 & 0.99 & 1.00 & 0.86 \\
\hline & $g_{3}$ & 1.00 & 1.00 & 1.00 & 0.99 & 1.00 & 1.00 & 0.50 \\
\hline & $g_{4}$ & 0.99 & 0.97 & 0.99 & 0.98 & 0.99 & 1.00 & 0.90 \\
\hline & $g_{5}$ & 0.99 & 1.00 & 0.99 & 0.97 & 0.99 & 1.00 & 0.74 \\
\hline & $g_{6}$ & 1.00 & 0.98 & 0.99 & 0.97 & 0.99 & 1.00 & 0.49 \\
\hline & $g_{7}$ & 1.00 & 0.99 & 0.99 & 0.98 & 1.00 & 1.00 & 0.06 \\
\hline \multirow[t]{7}{*}{3} & $g_{1}$ & 1.00 & 1.00 & 1.00 & 1.00 & 1.00 & 1.00 & 0.90 \\
\hline & $g_{2}$ & 1.00 & 1.00 & 1.00 & 1.00 & 1.00 & 1.00 & 0.87 \\
\hline & $g_{3}$ & 1.00 & 1.00 & 1.00 & 1.00 & 1.00 & 1.00 & 0.67 \\
\hline & $g_{4}$ & 1.00 & 1.00 & 1.00 & 1.00 & 1.00 & 1.00 & 0.90 \\
\hline & $g_{5}$ & 1.00 & 1.00 & 1.00 & 1.00 & 1.00 & 1.00 & 0.85 \\
\hline & $g_{6}$ & 1.00 & 0.99 & 1.00 & 0.99 & 1.00 & 1.00 & 0.67 \\
\hline & $g_{7}$ & 1.00 & 1.00 & 1.00 & 1.00 & 1.00 & 1.00 & 0.15 \\
\hline
\end{tabular}


Table 3: Results for $n=5$. Columns LRT and MCTs show estimation of the power of the particular tests. Columns BVS shows proportion of the (mean of) posterior probabilities of the null model given the data that are smaller then $\alpha=0.05,0.10,0.15$. Last column represent the estimated probability of the correct model having the highest mean of the posterior probability among all the possible models.

\begin{tabular}{|c|c|c|c|c|c|c|c|c|}
\hline$\lambda$ & Profile & LRT & $\begin{array}{r}\mathrm{MCT} \\
(\mathrm{W})\end{array}$ & $\begin{array}{r}\mathrm{MCT} \\
(\mathrm{M})\end{array}$ & $\begin{array}{l}\text { BVS } \\
0.05\end{array}$ & $\begin{array}{c}\text { BVS } \\
0.10\end{array}$ & $\begin{array}{l}\text { BVS } \\
0.15\end{array}$ & True $\mathrm{m}$. \\
\hline \multirow[t]{7}{*}{1} & $g_{1}$ & 0.55 & 0.62 & 0.54 & 0.31 & 0.45 & 0.55 & 0.62 \\
\hline & $g_{2}$ & 0.59 & 0.49 & 0.59 & 0.33 & 0.48 & 0.59 & 0.57 \\
\hline & $g_{3}$ & 0.66 & 0.66 & 0.64 & 0.37 & 0.52 & 0.65 & 0.01 \\
\hline & $g_{4}$ & 0.56 & 0.44 & 0.57 & 0.32 & 0.46 & 0.56 & 0.66 \\
\hline & $g_{5}$ & 0.63 & 0.64 & 0.59 & 0.32 & 0.48 & 0.61 & 0.08 \\
\hline & $g_{6}$ & 0.61 & 0.52 & 0.60 & 0.33 & 0.48 & 0.59 & 0.02 \\
\hline & $g_{7}$ & 0.64 & 0.62 & 0.61 & 0.36 & 0.50 & 0.62 & 0.00 \\
\hline \multirow[t]{7}{*}{1.5} & $g_{1}$ & 0.89 & 0.93 & 0.89 & 0.71 & 0.82 & 0.90 & 0.86 \\
\hline & $g_{2}$ & 0.90 & 0.81 & 0.90 & 0.74 & 0.85 & 0.91 & 0.83 \\
\hline & $g_{3}$ & 0.89 & 0.90 & 0.89 & 0.72 & 0.85 & 0.90 & 0.12 \\
\hline & $g_{4}$ & 0.88 & 0.77 & 0.88 & 0.70 & 0.81 & 0.87 & 0.87 \\
\hline & $g_{5}$ & 0.91 & 0.91 & 0.89 & 0.72 & 0.85 & 0.92 & 0.33 \\
\hline & $g_{6}$ & 0.90 & 0.82 & 0.90 & 0.74 & 0.85 & 0.91 & 0.16 \\
\hline & $g_{7}$ & 0.92 & 0.91 & 0.90 & 0.76 & 0.86 & 0.92 & 0.00 \\
\hline \multirow[t]{7}{*}{2} & $g_{1}$ & 0.99 & 1.00 & 0.99 & 0.93 & 0.97 & 0.99 & 0.91 \\
\hline & $g_{2}$ & 0.99 & 0.95 & 0.99 & 0.93 & 0.97 & 0.99 & 0.87 \\
\hline & $g_{3}$ & 0.99 & 0.99 & 0.99 & 0.94 & 0.98 & 0.99 & 0.37 \\
\hline & $g_{4}$ & 0.98 & 0.94 & 0.98 & 0.91 & 0.96 & 0.98 & 0.90 \\
\hline & $g_{5}$ & 0.99 & 0.99 & 0.98 & 0.94 & 0.97 & 0.99 & 0.60 \\
\hline & $g_{6}$ & 0.99 & 0.97 & 0.99 & 0.94 & 0.98 & 0.99 & 0.35 \\
\hline & $g_{7}$ & 1.00 & 0.99 & 0.99 & 0.95 & 0.99 & 1.00 & 0.02 \\
\hline \multirow[t]{7}{*}{2.5} & $g_{1}$ & 1.00 & 1.00 & 1.00 & 0.99 & 0.99 & 1.00 & 0.92 \\
\hline & $g_{2}$ & 1.00 & 1.00 & 1.00 & 0.99 & 1.00 & 1.00 & 0.90 \\
\hline & $g_{3}$ & 1.00 & 1.00 & 1.00 & 0.99 & 1.00 & 1.00 & 0.57 \\
\hline & $g_{4}$ & 1.00 & 0.99 & 1.00 & 0.99 & 1.00 & 1.00 & 0.90 \\
\hline & $g_{5}$ & 1.00 & 1.00 & 1.00 & 0.99 & 1.00 & 1.00 & 0.79 \\
\hline & $g_{6}$ & 1.00 & 1.00 & 1.00 & 1.00 & 1.00 & 1.00 & 0.59 \\
\hline & $g_{7}$ & 1.00 & 1.00 & 1.00 & 1.00 & 1.00 & 1.00 & 0.09 \\
\hline \multirow[t]{7}{*}{3} & $g_{1}$ & 1.00 & 1.00 & 1.00 & 1.00 & 1.00 & 1.00 & 0.93 \\
\hline & $g_{2}$ & 1.00 & 1.00 & 1.00 & 1.00 & 1.00 & 1.00 & 0.90 \\
\hline & $g_{3}$ & 1.00 & 1.00 & 1.00 & 1.00 & 1.00 & 1.00 & 0.79 \\
\hline & $g_{4}$ & 1.00 & 1.00 & 1.00 & 1.00 & 1.00 & 1.00 & 0.93 \\
\hline & $g_{5}$ & 1.00 & 1.00 & 1.00 & 1.00 & 1.00 & 1.00 & 0.90 \\
\hline & $g_{6}$ & 1.00 & 1.00 & 1.00 & 1.00 & 1.00 & 1.00 & 0.76 \\
\hline & $g_{7}$ & 1.00 & 1.00 & 1.00 & 1.00 & 1.00 & 1.00 & 0.23 \\
\hline
\end{tabular}


Table 4: Results for $K=5, n=3$, part 1. Columns LRT and MCTs show estimation of the power of the particular tests. Columns BVS shows proportion of the (mean of) posterior probabilities of the null model given the data that are smaller then $\alpha=0.05,0.10,0.15$. Last column represent the estimated probability of the correct model having the highest mean of the posterior probability among all the possible models.

\begin{tabular}{|c|c|c|c|c|c|c|c|c|}
\hline$\lambda$ & Profile & LRT & $\begin{array}{r}\mathrm{MCT} \\
(\mathrm{W})\end{array}$ & $\begin{array}{r}\text { MCT } \\
(\mathrm{M})\end{array}$ & $\begin{array}{l}\text { BVS } \\
0.05\end{array}$ & $\begin{array}{c}\text { BVS } \\
0.10\end{array}$ & $\begin{array}{r}\text { BVS } \\
0.15\end{array}$ & True m. \\
\hline \multirow[t]{15}{*}{1} & $g_{1}$ & 0.34 & 0.44 & 0.34 & 0.25 & 0.40 & 0.53 & 0.46 \\
\hline & $g_{2}$ & 0.35 & 0.29 & 0.34 & 0.25 & 0.39 & 0.51 & 0.40 \\
\hline & $g_{3}$ & 0.39 & 0.40 & 0.35 & 0.24 & 0.39 & 0.52 & 0.00 \\
\hline & $g_{4}$ & 0.35 & 0.25 & 0.32 & 0.22 & 0.39 & 0.52 & 0.39 \\
\hline & $g_{5}$ & 0.38 & 0.41 & 0.36 & 0.23 & 0.42 & 0.55 & 0.01 \\
\hline & $g_{6}$ & 0.48 & 0.37 & 0.43 & 0.32 & 0.49 & 0.60 & 0.00 \\
\hline & $g_{7}$ & 0.44 & 0.41 & 0.40 & 0.26 & 0.41 & 0.54 & 0.00 \\
\hline & $g_{8}$ & 0.36 & 0.28 & 0.36 & 0.26 & 0.41 & 0.52 & 0.52 \\
\hline & $g_{9}$ & 0.41 & 0.42 & 0.37 & 0.28 & 0.43 & 0.58 & 0.05 \\
\hline & $g_{10}$ & 0.42 & 0.34 & 0.36 & 0.26 & 0.40 & 0.54 & 0.02 \\
\hline & $g_{11}$ & 0.42 & 0.41 & 0.38 & 0.26 & 0.42 & 0.54 & 0.00 \\
\hline & $g_{12}$ & 0.40 & 0.28 & 0.37 & 0.22 & 0.39 & 0.50 & 0.01 \\
\hline & $g_{13}$ & 0.46 & 0.42 & 0.41 & 0.28 & 0.44 & 0.54 & 0.00 \\
\hline & $g_{14}$ & 0.48 & 0.38 & 0.43 & 0.27 & 0.43 & 0.57 & 0.00 \\
\hline & $g_{15}$ & 0.46 & 0.40 & 0.41 & 0.24 & 0.40 & 0.55 & 0.00 \\
\hline \multirow[t]{15}{*}{1.5} & $g_{1}$ & 0.63 & 0.73 & 0.62 & 0.52 & 0.70 & 0.80 & 0.71 \\
\hline & $g_{2}$ & 0.65 & 0.54 & 0.63 & 0.56 & 0.71 & 0.80 & 0.61 \\
\hline & $g_{3}$ & 0.66 & 0.67 & 0.62 & 0.54 & 0.72 & 0.82 & 0.03 \\
\hline & $g_{4}$ & 0.64 & 0.48 & 0.62 & 0.53 & 0.71 & 0.80 & 0.62 \\
\hline & $g_{5}$ & 0.70 & 0.72 & 0.67 & 0.61 & 0.77 & 0.85 & 0.09 \\
\hline & $g_{6}$ & 0.78 & 0.65 & 0.75 & 0.69 & 0.84 & 0.90 & 0.04 \\
\hline & $g_{7}$ & 0.72 & 0.70 & 0.68 & 0.62 & 0.77 & 0.86 & 0.00 \\
\hline & $g_{8}$ & 0.63 & 0.50 & 0.63 & 0.55 & 0.70 & 0.82 & 0.75 \\
\hline & $g_{9}$ & 0.69 & 0.72 & 0.66 & 0.60 & 0.76 & 0.84 & 0.22 \\
\hline & $g_{10}$ & 0.69 & 0.58 & 0.65 & 0.58 & 0.77 & 0.86 & 0.10 \\
\hline & $g_{11}$ & 0.72 & 0.71 & 0.66 & 0.61 & 0.79 & 0.88 & 0.00 \\
\hline & $g_{12}$ & 0.65 & 0.50 & 0.61 & 0.54 & 0.70 & 0.79 & 0.06 \\
\hline & $g_{13}$ & 0.73 & 0.69 & 0.69 & 0.62 & 0.78 & 0.87 & 0.00 \\
\hline & $g_{14}$ & 0.74 & 0.59 & 0.69 & 0.61 & 0.78 & 0.86 & 0.00 \\
\hline & $g_{15}$ & 0.73 & 0.67 & 0.67 & 0.60 & 0.75 & 0.85 & 0.00 \\
\hline \multirow[t]{7}{*}{2} & $g_{1}$ & 0.83 & 0.89 & 0.81 & 0.76 & 0.87 & 0.93 & 0.82 \\
\hline & $g_{2}$ & 0.86 & 0.76 & 0.85 & 0.81 & 0.91 & 0.94 & 0.76 \\
\hline & $g_{3}$ & 0.88 & 0.89 & 0.84 & 0.82 & 0.93 & 0.96 & 0.13 \\
\hline & $g_{4}$ & 0.87 & 0.71 & 0.86 & 0.82 & 0.92 & 0.96 & 0.73 \\
\hline & $g_{5}$ & 0.89 & 0.89 & 0.86 & 0.83 & 0.94 & 0.97 & 0.25 \\
\hline & $g_{6}$ & 0.94 & 0.86 & 0.92 & 0.91 & 0.96 & 0.98 & 0.13 \\
\hline & $g_{7}$ & 0.93 & 0.90 & 0.90 & 0.88 & 0.96 & 0.98 & 0.00 \\
\hline
\end{tabular}


Table 5: Results for $K=5, n=3$, part 2. Columns LRT and MCTs show estimation of the power of the particular tests. Columns BVS shows proportion of the (mean of) posterior probabilities of the null model given the data that are smaller then $\alpha=0.05,0.10,0.15$. Last column represent the estimated probability of the correct model having the highest mean of the posterior probability among all the possible models.

\begin{tabular}{|c|c|c|c|c|c|c|c|c|}
\hline$\lambda$ & Profile & LRT & $\begin{array}{r}\mathrm{MCT} \\
(\mathrm{W})\end{array}$ & $\begin{array}{r}\mathrm{MCT} \\
(\mathrm{M})\end{array}$ & $\begin{array}{r}\text { BVS } \\
0.05\end{array}$ & $\begin{array}{r}\text { BVS } \\
0.10\end{array}$ & $\begin{array}{r}\text { BVS } \\
0.15\end{array}$ & True m. \\
\hline \multirow[t]{8}{*}{2} & $g_{8}$ & 0.85 & 0.70 & 0.83 & 0.78 & 0.90 & 0.94 & 0.83 \\
\hline & $g_{9}$ & 0.88 & 0.91 & 0.86 & 0.82 & 0.92 & 0.95 & 0.43 \\
\hline & $g_{10}$ & 0.89 & 0.78 & 0.85 & 0.82 & 0.92 & 0.96 & 0.27 \\
\hline & $g_{11}$ & 0.92 & 0.90 & 0.88 & 0.86 & 0.95 & 0.98 & 0.01 \\
\hline & $g_{12}$ & 0.89 & 0.74 & 0.86 & 0.83 & 0.94 & 0.97 & 0.16 \\
\hline & $g_{13}$ & 0.90 & 0.87 & 0.86 & 0.84 & 0.94 & 0.97 & 0.01 \\
\hline & $g_{14}$ & 0.91 & 0.80 & 0.88 & 0.86 & 0.95 & 0.98 & 0.00 \\
\hline & $g_{15}$ & 0.91 & 0.87 & 0.86 & 0.86 & 0.94 & 0.97 & 0.00 \\
\hline \multirow[t]{15}{*}{2.5} & $g_{1}$ & 0.96 & 0.98 & 0.95 & 0.93 & 0.98 & 0.99 & 0.86 \\
\hline & $g_{2}$ & 0.95 & 0.89 & 0.94 & 0.93 & 0.97 & 0.99 & 0.78 \\
\hline & $g_{3}$ & 0.98 & 0.98 & 0.97 & 0.96 & 0.99 & 1.00 & 0.28 \\
\hline & $g_{4}$ & 0.97 & 0.86 & 0.97 & 0.95 & 0.98 & 0.99 & 0.78 \\
\hline & $g_{5}$ & 0.97 & 0.97 & 0.96 & 0.95 & 0.99 & 1.00 & 0.42 \\
\hline & $g_{6}$ & 0.99 & 0.95 & 0.99 & 0.99 & 1.00 & 1.00 & 0.28 \\
\hline & $g_{7}$ & 0.99 & 0.98 & 0.97 & 0.97 & 1.00 & 1.00 & 0.01 \\
\hline & $g_{8}$ & 0.95 & 0.87 & 0.94 & 0.92 & 0.97 & 0.99 & 0.86 \\
\hline & $g_{9}$ & 0.97 & 0.98 & 0.96 & 0.95 & 0.98 & 0.99 & 0.62 \\
\hline & $g_{10}$ & 0.98 & 0.94 & 0.97 & 0.97 & 0.99 & 1.00 & 0.45 \\
\hline & $g_{11}$ & 0.98 & 0.98 & 0.97 & 0.97 & 0.99 & 1.00 & 0.03 \\
\hline & $g_{12}$ & 0.98 & 0.91 & 0.97 & 0.97 & 0.99 & 1.00 & 0.31 \\
\hline & $g_{13}$ & 0.98 & 0.97 & 0.97 & 0.97 & 0.99 & 1.00 & 0.05 \\
\hline & $g_{14}$ & 0.99 & 0.94 & 0.97 & 0.98 & 1.00 & 1.00 & 0.01 \\
\hline & $g_{15}$ & 0.99 & 0.98 & 0.97 & 0.98 & 0.99 & 1.00 & 0.00 \\
\hline \multirow[t]{15}{*}{3} & $g_{1}$ & 0.99 & 1.00 & 0.99 & 0.99 & 1.00 & 1.00 & 0.88 \\
\hline & $g_{2}$ & 0.99 & 0.97 & 0.99 & 0.99 & 1.00 & 1.00 & 0.78 \\
\hline & $g_{3}$ & 1.00 & 1.00 & 0.99 & 0.99 & 1.00 & 1.00 & 0.46 \\
\hline & $g_{4}$ & 1.00 & 0.94 & 0.99 & 0.99 & 1.00 & 1.00 & 0.81 \\
\hline & $g_{5}$ & 0.99 & 0.99 & 0.99 & 0.99 & 1.00 & 1.00 & 0.61 \\
\hline & $g_{6}$ & 1.00 & 0.99 & 1.00 & 1.00 & 1.00 & 1.00 & 0.46 \\
\hline & $g_{7}$ & 1.00 & 0.99 & 0.99 & 1.00 & 1.00 & 1.00 & 0.04 \\
\hline & $g_{8}$ & 0.99 & 0.95 & 0.99 & 0.98 & 0.99 & 1.00 & 0.86 \\
\hline & $g_{9}$ & 1.00 & 1.00 & 0.99 & 0.99 & 1.00 & 1.00 & 0.77 \\
\hline & $g_{10}$ & 1.00 & 0.99 & 1.00 & 0.99 & 1.00 & 1.00 & 0.61 \\
\hline & $g_{11}$ & 1.00 & 1.00 & 1.00 & 1.00 & 1.00 & 1.00 & 0.10 \\
\hline & $g_{12}$ & 1.00 & 0.97 & 0.99 & 0.99 & 1.00 & 1.00 & 0.48 \\
\hline & $g_{13}$ & 1.00 & 0.99 & 0.99 & 0.99 & 1.00 & 1.00 & 0.12 \\
\hline & $g_{14}$ & 1.00 & 0.99 & 1.00 & 1.00 & 1.00 & 1.00 & 0.04 \\
\hline & $g_{15}$ & 1.00 & 0.99 & 1.00 & 1.00 & 1.00 & 1.00 & 0.00 \\
\hline
\end{tabular}


Table 6: Results for $K=5, n=4$, part 1. Columns LRT and MCTs show estimation of the power of the particular tests. Columns BVS shows proportion of the (mean of) posterior probabilities of the null model given the data that are smaller then $\alpha=0.05,0.10,0.15$. Last column represent the estimated probability of the correct model having the highest mean of the posterior probability among all the possible models.

\begin{tabular}{|c|c|c|c|c|c|c|c|c|}
\hline$\lambda$ & Profile & LRT & $\begin{array}{r}\mathrm{MCT} \\
(\mathrm{W})\end{array}$ & $\begin{array}{r}\mathrm{MCT} \\
(\mathrm{M})\end{array}$ & $\begin{array}{l}\text { BVS } \\
0.05\end{array}$ & $\begin{array}{r}\text { BVS } \\
0.10\end{array}$ & $\begin{array}{r}\text { BVS } \\
0.15\end{array}$ & True $m$. \\
\hline \multirow[t]{15}{*}{1} & $g_{1}$ & 0.44 & 0.54 & 0.44 & 0.28 & 0.42 & 0.54 & 0.52 \\
\hline & $g_{2}$ & 0.48 & 0.39 & 0.46 & 0.30 & 0.46 & 0.57 & 0.45 \\
\hline & $g_{3}$ & 0.49 & 0.51 & 0.47 & 0.29 & 0.45 & 0.56 & 0.00 \\
\hline & $g_{4}$ & 0.47 & 0.33 & 0.45 & 0.30 & 0.44 & 0.54 & 0.49 \\
\hline & $g_{5}$ & 0.56 & 0.55 & 0.52 & 0.34 & 0.50 & 0.61 & 0.02 \\
\hline & $g_{6}$ & 0.61 & 0.49 & 0.59 & 0.41 & 0.55 & 0.67 & 0.01 \\
\hline & $g_{7}$ & 0.57 & 0.54 & 0.54 & 0.33 & 0.51 & 0.62 & 0.00 \\
\hline & $g_{8}$ & 0.44 & 0.36 & 0.44 & 0.30 & 0.43 & 0.54 & 0.58 \\
\hline & $g_{9}$ & 0.52 & 0.55 & 0.49 & 0.31 & 0.47 & 0.60 & 0.08 \\
\hline & $g_{10}$ & 0.56 & 0.45 & 0.52 & 0.33 & 0.49 & 0.61 & 0.03 \\
\hline & $g_{11}$ & 0.56 & 0.54 & 0.53 & 0.33 & 0.50 & 0.61 & 0.00 \\
\hline & $g_{12}$ & 0.49 & 0.37 & 0.46 & 0.28 & 0.42 & 0.54 & 0.01 \\
\hline & $g_{13}$ & 0.54 & 0.50 & 0.50 & 0.30 & 0.47 & 0.57 & 0.00 \\
\hline & $g_{14}$ & 0.55 & 0.42 & 0.52 & 0.31 & 0.46 & 0.56 & 0.00 \\
\hline & $g_{15}$ & 0.53 & 0.49 & 0.50 & 0.28 & 0.44 & 0.56 & 0.00 \\
\hline \multirow[t]{15}{*}{1.5} & $g_{1}$ & 0.78 & 0.85 & 0.77 & 0.62 & 0.78 & 0.85 & 0.80 \\
\hline & $g_{2}$ & 0.79 & 0.68 & 0.78 & 0.65 & 0.81 & 0.87 & 0.69 \\
\hline & $g_{3}$ & 0.82 & 0.84 & 0.79 & 0.66 & 0.81 & 0.88 & 0.04 \\
\hline & $g_{4}$ & 0.77 & 0.60 & 0.76 & 0.65 & 0.78 & 0.85 & 0.69 \\
\hline & $g_{5}$ & 0.83 & 0.82 & 0.81 & 0.68 & 0.83 & 0.90 & 0.10 \\
\hline & $g_{6}$ & 0.90 & 0.78 & 0.89 & 0.80 & 0.90 & 0.93 & 0.06 \\
\hline & $g_{7}$ & 0.85 & 0.83 & 0.83 & 0.72 & 0.85 & 0.90 & 0.00 \\
\hline & $g_{8}$ & 0.77 & 0.63 & 0.76 & 0.63 & 0.77 & 0.84 & 0.81 \\
\hline & $g_{9}$ & 0.83 & 0.85 & 0.80 & 0.67 & 0.83 & 0.88 & 0.24 \\
\hline & $g_{10}$ & 0.82 & 0.72 & 0.79 & 0.67 & 0.81 & 0.88 & 0.17 \\
\hline & $g_{11}$ & 0.82 & 0.82 & 0.78 & 0.68 & 0.81 & 0.88 & 0.00 \\
\hline & $g_{12}$ & 0.81 & 0.67 & 0.79 & 0.68 & 0.81 & 0.87 & 0.06 \\
\hline & $g_{13}$ & 0.86 & 0.84 & 0.84 & 0.72 & 0.85 & 0.91 & 0.00 \\
\hline & $g_{14}$ & 0.83 & 0.70 & 0.81 & 0.68 & 0.82 & 0.88 & 0.00 \\
\hline & $g_{15}$ & 0.83 & 0.79 & 0.80 & 0.67 & 0.81 & 0.89 & 0.00 \\
\hline \multirow[t]{7}{*}{2} & $g_{1}$ & 0.94 & 0.97 & 0.95 & 0.89 & 0.95 & 0.97 & 0.88 \\
\hline & $g_{2}$ & 0.94 & 0.87 & 0.94 & 0.88 & 0.94 & 0.97 & 0.81 \\
\hline & $g_{3}$ & 0.95 & 0.96 & 0.94 & 0.90 & 0.96 & 0.97 & 0.17 \\
\hline & $g_{4}$ & 0.96 & 0.82 & 0.94 & 0.87 & 0.95 & 0.97 & 0.80 \\
\hline & $g_{5}$ & 0.97 & 0.97 & 0.96 & 0.90 & 0.97 & 0.99 & 0.30 \\
\hline & $g_{6}$ & 0.98 & 0.95 & 0.98 & 0.96 & 0.99 & 0.99 & 0.21 \\
\hline & $g_{7}$ & 0.97 & 0.96 & 0.96 & 0.93 & 0.98 & 0.99 & 0.00 \\
\hline
\end{tabular}


Table 7: Results for $K=5, n=4$, part 2.Columns LRT and MCTs show estimation of the power of the particular tests. Columns BVS shows proportion of the (mean of) posterior probabilities of the null model given the data that are smaller then $\alpha=0.05,0.10,0.15$. Last column represent the estimated probability of the correct model having the highest mean of the posterior probability among all the possible models.

\begin{tabular}{|c|c|c|c|c|c|c|c|c|}
\hline$\lambda$ & Profile & LRT & $\begin{array}{r}\mathrm{MCT} \\
(\mathrm{W})\end{array}$ & $\begin{array}{r}\mathrm{MCT} \\
(\mathrm{M})\end{array}$ & $\begin{array}{r}\text { BVS } \\
0.05\end{array}$ & $\begin{array}{r}\text { BVS } \\
0.10\end{array}$ & $\begin{array}{r}\text { BVS } \\
0.15\end{array}$ & True m. \\
\hline \multirow[t]{8}{*}{2} & $g_{8}$ & 0.96 & 0.86 & 0.96 & 0.90 & 0.95 & 0.98 & 0.87 \\
\hline & $g_{9}$ & 0.96 & 0.97 & 0.95 & 0.90 & 0.95 & 0.98 & 0.50 \\
\hline & $g_{10}$ & 0.96 & 0.91 & 0.96 & 0.91 & 0.97 & 0.98 & 0.34 \\
\hline & $g_{11}$ & 0.97 & 0.96 & 0.95 & 0.92 & 0.97 & 0.99 & 0.02 \\
\hline & $g_{12}$ & 0.96 & 0.87 & 0.95 & 0.90 & 0.96 & 0.98 & 0.19 \\
\hline & $g_{13}$ & 0.96 & 0.95 & 0.95 & 0.92 & 0.97 & 0.99 & 0.02 \\
\hline & $g_{14}$ & 0.97 & 0.91 & 0.96 & 0.94 & 0.97 & 0.99 & 0.00 \\
\hline & $g_{15}$ & 0.98 & 0.96 & 0.97 & 0.94 & 0.98 & 0.99 & 0.00 \\
\hline \multirow[t]{15}{*}{2.5} & $g_{1}$ & 0.99 & 1.00 & 0.99 & 0.98 & 0.99 & 1.00 & 0.88 \\
\hline & $g_{2}$ & 0.99 & 0.97 & 0.99 & 0.98 & 1.00 & 1.00 & 0.82 \\
\hline & $g_{3}$ & 0.99 & 0.99 & 0.99 & 0.98 & 1.00 & 1.00 & 0.36 \\
\hline & $g_{4}$ & 0.99 & 0.95 & 0.99 & 0.98 & 0.99 & 1.00 & 0.82 \\
\hline & $g_{5}$ & 1.00 & 1.00 & 1.00 & 0.99 & 1.00 & 1.00 & 0.56 \\
\hline & $g_{6}$ & 1.00 & 1.00 & 1.00 & 1.00 & 1.00 & 1.00 & 0.40 \\
\hline & $g_{7}$ & 1.00 & 1.00 & 1.00 & 0.99 & 1.00 & 1.00 & 0.02 \\
\hline & $g_{8}$ & 0.99 & 0.96 & 0.99 & 0.98 & 0.99 & 1.00 & 0.87 \\
\hline & $g_{9}$ & 0.99 & 1.00 & 0.99 & 0.97 & 0.99 & 1.00 & 0.73 \\
\hline & $g_{10}$ & 1.00 & 0.98 & 1.00 & 0.99 & 1.00 & 1.00 & 0.55 \\
\hline & $g_{11}$ & 1.00 & 1.00 & 1.00 & 0.99 & 1.00 & 1.00 & 0.06 \\
\hline & $g_{12}$ & 0.99 & 0.96 & 0.99 & 0.98 & 0.99 & 1.00 & 0.40 \\
\hline & $g_{13}$ & 1.00 & 0.99 & 1.00 & 0.99 & 1.00 & 1.00 & 0.06 \\
\hline & $g_{14}$ & 1.00 & 0.99 & 1.00 & 0.99 & 1.00 & 1.00 & 0.02 \\
\hline & $g_{15}$ & 1.00 & 0.99 & 1.00 & 0.99 & 1.00 & 1.00 & 0.00 \\
\hline \multirow[t]{15}{*}{3} & $g_{1}$ & 1.00 & 1.00 & 1.00 & 1.00 & 1.00 & 1.00 & 0.89 \\
\hline & $g_{2}$ & 1.00 & 1.00 & 1.00 & 1.00 & 1.00 & 1.00 & 0.85 \\
\hline & $g_{3}$ & 1.00 & 1.00 & 1.00 & 1.00 & 1.00 & 1.00 & 0.59 \\
\hline & $g_{4}$ & 1.00 & 0.99 & 1.00 & 1.00 & 1.00 & 1.00 & 0.86 \\
\hline & $g_{5}$ & 1.00 & 1.00 & 1.00 & 1.00 & 1.00 & 1.00 & 0.71 \\
\hline & $g_{6}$ & 1.00 & 1.00 & 1.00 & 1.00 & 1.00 & 1.00 & 0.57 \\
\hline & $g_{7}$ & 1.00 & 1.00 & 1.00 & 1.00 & 1.00 & 1.00 & 0.07 \\
\hline & $g_{8}$ & 1.00 & 0.99 & 1.00 & 1.00 & 1.00 & 1.00 & 0.89 \\
\hline & $g_{9}$ & 1.00 & 1.00 & 1.00 & 1.00 & 1.00 & 1.00 & 0.85 \\
\hline & $g_{10}$ & 1.00 & 1.00 & 1.00 & 1.00 & 1.00 & 1.00 & 0.69 \\
\hline & $g_{11}$ & 1.00 & 1.00 & 1.00 & 1.00 & 1.00 & 1.00 & 0.12 \\
\hline & $g_{12}$ & 1.00 & 1.00 & 1.00 & 1.00 & 1.00 & 1.00 & 0.58 \\
\hline & $g_{13}$ & 1.00 & 1.00 & 1.00 & 1.00 & 1.00 & 1.00 & 0.16 \\
\hline & $g_{14}$ & 1.00 & 1.00 & 1.00 & 1.00 & 1.00 & 1.00 & 0.07 \\
\hline & $g_{15}$ & 1.00 & 1.00 & 1.00 & 1.00 & 1.00 & 1.00 & 0.00 \\
\hline
\end{tabular}


Table 8: Results for $K=5, n=5$, part 1. Columns LRT and MCTs show estimation of the power of the particular tests. Columns BVS shows proportion of the (mean of) posterior probabilities of the null model given the data that are smaller then $\alpha=0.05,0.10,0.15$. Last column represent the estimated probability of the correct model having the highest mean of the posterior probability among all the possible models.

\begin{tabular}{|c|c|c|c|c|c|c|c|c|}
\hline$\lambda$ & Profile & LRT & $\begin{array}{r}\mathrm{MCT} \\
(\mathrm{W})\end{array}$ & $\begin{array}{r}\text { MCT } \\
(\mathrm{M})\end{array}$ & $\begin{array}{l}\text { BVS } \\
0.05\end{array}$ & $\begin{array}{c}\text { BVS } \\
0.10\end{array}$ & $\begin{array}{r}\text { BVS } \\
0.15\end{array}$ & True m. \\
\hline \multirow[t]{15}{*}{1} & $g_{1}$ & 0.54 & 0.65 & 0.53 & 0.31 & 0.46 & 0.58 & 0.62 \\
\hline & $g_{2}$ & 0.56 & 0.46 & 0.55 & 0.35 & 0.49 & 0.59 & 0.51 \\
\hline & $g_{3}$ & 0.60 & 0.63 & 0.58 & 0.36 & 0.52 & 0.62 & 0.00 \\
\hline & $g_{4}$ & 0.57 & 0.41 & 0.57 & 0.35 & 0.50 & 0.60 & 0.53 \\
\hline & $g_{5}$ & 0.62 & 0.63 & 0.60 & 0.40 & 0.54 & 0.64 & 0.02 \\
\hline & $g_{6}$ & 0.68 & 0.56 & 0.66 & 0.43 & 0.59 & 0.70 & 0.00 \\
\hline & $g_{7}$ & 0.64 & 0.59 & 0.61 & 0.34 & 0.50 & 0.64 & 0.00 \\
\hline & $g_{8}$ & 0.58 & 0.47 & 0.58 & 0.37 & 0.52 & 0.61 & 0.63 \\
\hline & $g_{9}$ & 0.59 & 0.63 & 0.56 & 0.35 & 0.50 & 0.61 & 0.07 \\
\hline & $g_{10}$ & 0.59 & 0.50 & 0.57 & 0.34 & 0.50 & 0.61 & 0.03 \\
\hline & $g_{11}$ & 0.64 & 0.62 & 0.60 & 0.35 & 0.53 & 0.64 & 0.00 \\
\hline & $g_{12}$ & 0.59 & 0.43 & 0.58 & 0.33 & 0.49 & 0.59 & 0.01 \\
\hline & $g_{13}$ & 0.63 & 0.59 & 0.61 & 0.39 & 0.53 & 0.63 & 0.00 \\
\hline & $g_{14}$ & 0.65 & 0.51 & 0.63 & 0.36 & 0.52 & 0.64 & 0.00 \\
\hline & $g_{15}$ & 0.64 & 0.60 & 0.60 & 0.35 & 0.50 & 0.61 & 0.00 \\
\hline \multirow[t]{15}{*}{1.5} & $g_{1}$ & 0.85 & 0.92 & 0.86 & 0.72 & 0.83 & 0.89 & 0.81 \\
\hline & $g_{2}$ & 0.88 & 0.76 & 0.87 & 0.75 & 0.84 & 0.90 & 0.75 \\
\hline & $g_{3}$ & 0.88 & 0.88 & 0.86 & 0.74 & 0.86 & 0.91 & 0.07 \\
\hline & $g_{4}$ & 0.85 & 0.69 & 0.84 & 0.71 & 0.82 & 0.88 & 0.73 \\
\hline & $g_{5}$ & 0.89 & 0.89 & 0.87 & 0.75 & 0.86 & 0.91 & 0.16 \\
\hline & $g_{6}$ & 0.94 & 0.86 & 0.94 & 0.85 & 0.93 & 0.96 & 0.07 \\
\hline & $g_{7}$ & 0.92 & 0.91 & 0.91 & 0.80 & 0.89 & 0.94 & 0.00 \\
\hline & $g_{8}$ & 0.86 & 0.70 & 0.86 & 0.71 & 0.83 & 0.87 & 0.82 \\
\hline & $g_{9}$ & 0.90 & 0.93 & 0.89 & 0.76 & 0.86 & 0.92 & 0.34 \\
\hline & $g_{10}$ & 0.91 & 0.83 & 0.90 & 0.79 & 0.88 & 0.93 & 0.18 \\
\hline & $g_{11}$ & 0.90 & 0.90 & 0.88 & 0.77 & 0.88 & 0.92 & 0.00 \\
\hline & $g_{12}$ & 0.89 & 0.76 & 0.88 & 0.74 & 0.85 & 0.92 & 0.07 \\
\hline & $g_{13}$ & 0.90 & 0.90 & 0.89 & 0.78 & 0.87 & 0.93 & 0.00 \\
\hline & $g_{14}$ & 0.92 & 0.82 & 0.90 & 0.79 & 0.89 & 0.94 & 0.00 \\
\hline & $g_{15}$ & 0.91 & 0.88 & 0.89 & 0.76 & 0.88 & 0.93 & 0.00 \\
\hline \multirow[t]{7}{*}{2} & $g_{1}$ & 0.98 & 0.99 & 0.98 & 0.93 & 0.97 & 0.98 & 0.91 \\
\hline & $g_{2}$ & 0.98 & 0.94 & 0.99 & 0.94 & 0.98 & 0.99 & 0.86 \\
\hline & $g_{3}$ & 0.99 & 0.99 & 0.99 & 0.95 & 0.99 & 0.99 & 0.23 \\
\hline & $g_{4}$ & 0.98 & 0.91 & 0.99 & 0.95 & 0.98 & 0.99 & 0.84 \\
\hline & $g_{5}$ & 0.99 & 0.99 & 0.99 & 0.96 & 0.98 & 0.99 & 0.40 \\
\hline & $g_{6}$ & 1.00 & 0.98 & 1.00 & 0.99 & 1.00 & 1.00 & 0.24 \\
\hline & $g_{7}$ & 0.99 & 0.99 & 0.99 & 0.96 & 0.99 & 0.99 & 0.00 \\
\hline
\end{tabular}


Table 9: Results for $K=5, n=5$, part 2. Columns LRT and MCTs show estimation of the power of the particular tests. Columns BVS shows proportion of the (mean of) posterior probabilities of the null model given the data that are smaller then $\alpha=0.05,0.10,0.15$. Last column represent the estimated probability of the correct model having the highest mean of the posterior probability among all the possible models.

\begin{tabular}{|c|c|c|c|c|c|c|c|c|}
\hline$\lambda$ & Profile & LRT & $\begin{array}{r}\mathrm{MCT} \\
(\mathrm{W})\end{array}$ & $\begin{array}{r}\mathrm{MCT} \\
(\mathrm{M})\end{array}$ & $\begin{array}{r}\text { BVS } \\
0.05\end{array}$ & $\begin{array}{c}\text { BVS } \\
0.10\end{array}$ & $\begin{array}{r}\text { BVS } \\
0.15\end{array}$ & True m. \\
\hline \multirow[t]{8}{*}{2} & $g_{8}$ & 0.99 & 0.94 & 0.99 & 0.95 & 0.98 & 0.99 & 0.90 \\
\hline & $g_{9}$ & 0.98 & 0.99 & 0.98 & 0.94 & 0.97 & 0.99 & 0.63 \\
\hline & $g_{10}$ & 0.99 & 0.97 & 0.99 & 0.95 & 0.98 & 0.99 & 0.42 \\
\hline & $g_{11}$ & 0.99 & 0.99 & 0.99 & 0.96 & 0.98 & 1.00 & 0.02 \\
\hline & $g_{12}$ & 0.99 & 0.94 & 0.99 & 0.95 & 0.99 & 0.99 & 0.26 \\
\hline & $g_{13}$ & 0.99 & 0.99 & 0.99 & 0.96 & 0.99 & 1.00 & 0.02 \\
\hline & $g_{14}$ & 0.99 & 0.97 & 0.99 & 0.97 & 0.99 & 0.99 & 0.00 \\
\hline & $g_{15}$ & 0.99 & 0.99 & 0.99 & 0.97 & 0.99 & 0.99 & 0.00 \\
\hline \multirow[t]{15}{*}{2.5} & $g_{1}$ & 1.00 & 1.00 & 1.00 & 1.00 & 1.00 & 1.00 & 0.90 \\
\hline & $g_{2}$ & 1.00 & 0.99 & 1.00 & 0.99 & 1.00 & 1.00 & 0.88 \\
\hline & $g_{3}$ & 1.00 & 1.00 & 1.00 & 1.00 & 1.00 & 1.00 & 0.44 \\
\hline & $g_{4}$ & 1.00 & 0.99 & 1.00 & 1.00 & 1.00 & 1.00 & 0.85 \\
\hline & $g_{5}$ & 1.00 & 1.00 & 1.00 & 0.99 & 1.00 & 1.00 & 0.60 \\
\hline & $g_{6}$ & 1.00 & 1.00 & 1.00 & 1.00 & 1.00 & 1.00 & 0.50 \\
\hline & $g_{7}$ & 1.00 & 1.00 & 1.00 & 0.99 & 1.00 & 1.00 & 0.02 \\
\hline & $g_{8}$ & 1.00 & 0.99 & 1.00 & 0.99 & 1.00 & 1.00 & 0.90 \\
\hline & $g_{9}$ & 1.00 & 1.00 & 1.00 & 1.00 & 1.00 & 1.00 & 0.81 \\
\hline & $g_{10}$ & 1.00 & 0.99 & 1.00 & 1.00 & 1.00 & 1.00 & 0.68 \\
\hline & $g_{11}$ & 1.00 & 1.00 & 1.00 & 1.00 & 1.00 & 1.00 & 0.08 \\
\hline & $g_{12}$ & 1.00 & 0.99 & 1.00 & 1.00 & 1.00 & 1.00 & 0.49 \\
\hline & $g_{13}$ & 1.00 & 1.00 & 1.00 & 1.00 & 1.00 & 1.00 & 0.09 \\
\hline & $g_{14}$ & 1.00 & 1.00 & 1.00 & 1.00 & 1.00 & 1.00 & 0.03 \\
\hline & $g_{15}$ & 1.00 & 1.00 & 1.00 & 1.00 & 1.00 & 1.00 & 0.00 \\
\hline \multirow[t]{15}{*}{3} & $g_{1}$ & 1.00 & 1.00 & 1.00 & 1.00 & 1.00 & 1.00 & 0.91 \\
\hline & $g_{2}$ & 1.00 & 1.00 & 1.00 & 1.00 & 1.00 & 1.00 & 0.88 \\
\hline & $g_{3}$ & 1.00 & 1.00 & 1.00 & 1.00 & 1.00 & 1.00 & 0.64 \\
\hline & $g_{4}$ & 1.00 & 1.00 & 1.00 & 1.00 & 1.00 & 1.00 & 0.87 \\
\hline & $g_{5}$ & 1.00 & 1.00 & 1.00 & 1.00 & 1.00 & 1.00 & 0.80 \\
\hline & $g_{6}$ & 1.00 & 1.00 & 1.00 & 1.00 & 1.00 & 1.00 & 0.69 \\
\hline & $g_{7}$ & 1.00 & 1.00 & 1.00 & 1.00 & 1.00 & 1.00 & 0.09 \\
\hline & $g_{8}$ & 1.00 & 1.00 & 1.00 & 1.00 & 1.00 & 1.00 & 0.92 \\
\hline & $g_{9}$ & 1.00 & 1.00 & 1.00 & 1.00 & 1.00 & 1.00 & 0.88 \\
\hline & $g_{10}$ & 1.00 & 1.00 & 1.00 & 1.00 & 1.00 & 1.00 & 0.81 \\
\hline & $g_{11}$ & 1.00 & 1.00 & 1.00 & 1.00 & 1.00 & 1.00 & 0.23 \\
\hline & $g_{12}$ & 1.00 & 1.00 & 1.00 & 1.00 & 1.00 & 1.00 & 0.70 \\
\hline & $g_{13}$ & 1.00 & 1.00 & 1.00 & 1.00 & 1.00 & 1.00 & 0.24 \\
\hline & $g_{14}$ & 1.00 & 1.00 & 1.00 & 1.00 & 1.00 & 1.00 & 0.08 \\
\hline & $g_{15}$ & 1.00 & 1.00 & 1.00 & 1.00 & 1.00 & 1.00 & 0.00 \\
\hline
\end{tabular}




\section{LIKELIHOOD RATIO TEST}

The likelihood ratio test is discussed in Section 3.1 in the manuscript. The null distribution of $T_{L R T}$ is a mixture of Beta distributions with mixture probabilities $P(\ell, K, \boldsymbol{w}), \ell=1, \ldots, K$, that are also known as the level probabilities. They represent the probability (under the null hypothesis) that the number of unique isotonic means equals to $\ell$ in an experiment with $K$ possible levels. According to Barlow et al. (1972), the $p$-value can be calculated by

$$
P_{H_{0}}\left(T_{L R T} \geq t_{L R T}\right)=\sum_{\ell=1}^{K} P(\ell, K, \boldsymbol{w}) P\left[B_{\frac{1}{2}(\ell-1), \frac{1}{2}(N-\ell)} \geq t_{L R T}\right]
$$

with $N$ being the total number of observations, $\ell$ the number of final levels and $B_{\frac{1}{2}(\ell-1), \frac{1}{2}(N-\ell)}$ denotes a Beta distribution with $\alpha=1 / 2(\ell-1)$ and $\beta=1 / 2(N-\ell)$. The inverse $\boldsymbol{w}^{-1}=$ $\left(w_{0}^{-1}, \ldots, w_{K}^{-1}\right)$ equals the variance of the response at each dose. For example, for case of $K=4$ and equal weights $\boldsymbol{w}_{0}$, the probability for one level only equals $P\left(\ell=1,4, \boldsymbol{w}_{0}\right)=0.25$, $P\left(\ell=2,4, \boldsymbol{w}_{0}\right)=0.46, P\left(\ell=3,4, \boldsymbol{w}_{0}\right)=0.25$ and $P\left(\ell=4,4, \boldsymbol{w}_{0}\right)=0.04$ (Robertson et al. $1988)$. Note that level probabilities themselves are related to the isotonic regression results and not to the testing of the null hypothesis. They show the probability of obtaining certain number of the isotonic means under the null hypothesis. Hence, they do not depend on the variability of the data unless the variability differs across the doses.

\section{MULTIPLE CONTRAST TESTS}

In this section we discuss in more details the contrast matrices for the MCT introduced in Section 3.1 in the manuscript. The MCT for the set of the single contacts tests $\left(T_{1}^{S C}, T_{2}^{S C} \ldots T_{V}^{S C}\right)$ can be defined using a contrast matrix given by

$$
\boldsymbol{C}^{M C}=\left(\begin{array}{l}
\boldsymbol{c}_{1} \\
\boldsymbol{c}_{2} \\
\vdots \\
\boldsymbol{c}_{V}
\end{array}\right)=\left(\begin{array}{llll}
c_{10} & c_{11} & \ldots & c_{1, K-1} \\
c_{20} & c_{21} & \ldots & c_{2, K-1} \\
\vdots & & \vdots & \\
c_{V 0} & c_{V 1} & \ldots & c_{V K}
\end{array}\right)
$$

Each row of the contrast matrix $\boldsymbol{C}^{M C}$ corresponds to one contrast vector $\boldsymbol{c}$ of the SCT. There exist lot of useful choices of the contrast matrices (Hothorn 2006, e.g.). For the further comparison, we use two of them: Williams' and Marcus' MCTs (Bretz 1999) based on the tests designed by Williams (1971) and Marcus (1976). Designs of the tests determine the choice of $\boldsymbol{c}_{v}, v=1, \ldots, V$. Williams' MCT is based on the comparison between first (usually control) dose and the weighted average over the last $b(b=1, \ldots, K-1)$ doses. It originates from a comparison of the last dose mean $\hat{\mu}_{K-1}^{\star}$ computed using the isotonic regression with an estimate of the mean of the first dose $\hat{\mu}_{0}$ under the different possible profiles. Hence, due to properties of the PAVA algorithm it holds that

$$
\hat{\mu}_{K-1}^{\star}-\hat{\mu}_{0}=\max \boldsymbol{C}^{W i l} \hat{\boldsymbol{\mu}},
$$


where $\hat{\boldsymbol{\mu}}=\left(\hat{\mu}_{0}, \ldots \hat{\mu}_{K-1}\right)^{T}$ and

$$
C^{W i l}=\left(\begin{array}{ccccc}
-1 & 0 & \cdots & 0 & 1 \\
-1 & 0 & \cdots & \frac{n_{K-2}}{n_{K-2}+n_{K-1}} & \frac{n_{K-1}}{n_{K-2}+n_{K-1}} \\
\vdots & \vdots & \cdots & \vdots & \vdots \\
-1 & \frac{n_{1}}{n_{1}+\ldots+n_{K-1}} & \cdots & \frac{n_{K-2}}{n_{1}+\ldots+n_{K-1}} & \frac{n_{K-1}}{n_{1}+\ldots+n_{K-1}}
\end{array}\right) .
$$

The matrix $\boldsymbol{C}^{W i l}$ we call a Williams-type MCT matrix and we use it to construct our set of the MCTs through Equation (3).

Marcus' MCT is a modification of Williams' idea with replacing the estimate of the mean of the first dose $\hat{\mu}_{0}$ with the isotonic estimate $\hat{\mu}_{0}^{\star}$. Unfortunately, there is no general close form solution for $\boldsymbol{C}$ as in case of Williams, since its structure depends on the number of the doses. It can be easily constructed using each element of the following relationship as one contrast:

$$
\hat{\mu}_{K-1}^{\star}-\hat{\mu}_{0}^{\star}=\max \left\{0, \max _{0 \leq g, h \leq K-1}\left\{\frac{n_{g} \hat{\mu}_{g}+\ldots+n_{K-1} \hat{\mu}_{K-1}}{n_{g}+\ldots+n_{K-1}}-\frac{n_{0} \hat{\mu}_{0}+\ldots+n_{h} \hat{\mu}_{h}}{n_{0}+\ldots+n_{h}}\right\}\right\} .
$$

The inference of Williams' and Marcus' MCTs can be based on the multivariate $t$-distribution. For the details about the distribution and about both procedures, we recommend to see Lin et al. (2012).

\section{BAYESIAN VARIABLE SELECTION}

\subsection{Design Matrices}

As mentioned in the manuscript in Section 1 and Section 3, the BVS models fits all possible one-way ordered restricted ANOVA models for a given value of $K$. Each one of the models is defined using a model specific design matrix and a parameter vector. For example, for an experiment with $K=4$ dose levels, the design matrices for all the models presented in Table 1 are given, respectively, by 


$$
\begin{aligned}
& \boldsymbol{X}_{\left(g_{0}\right)}=\left(\begin{array}{c}
1 \\
1 \\
1 \\
1 \\
1 \\
1 \\
1 \\
1 \\
1 \\
1 \\
1 \\
1
\end{array}\right), \quad \boldsymbol{X}_{\left(g_{1}\right)}=\left(\begin{array}{cc}
1 & 0 \\
1 & 0 \\
1 & 0 \\
1 & 1 \\
1 & 1 \\
1 & 1 \\
1 & 1 \\
1 & 1 \\
1 & 1 \\
1 & 1 \\
1 & 1 \\
1 & 1
\end{array}\right), \boldsymbol{X}_{\left(g_{2}\right)}=\left(\begin{array}{ll}
1 & 0 \\
1 & 0 \\
1 & 0 \\
1 & 0 \\
1 & 0 \\
1 & 0 \\
1 & 1 \\
1 & 1 \\
1 & 1 \\
1 & 1 \\
1 & 1 \\
1 & 1
\end{array}\right), \\
& \boldsymbol{X}_{\left(g_{3}\right)}=\left(\begin{array}{ccc}
1 & 0 & 0 \\
1 & 0 & 0 \\
1 & 0 & 0 \\
1 & 1 & 0 \\
1 & 1 & 0 \\
1 & 1 & 0 \\
1 & 1 & 1 \\
1 & 1 & 1 \\
1 & 1 & 1 \\
1 & 1 & 1 \\
1 & 1 & 1 \\
1 & 1 & 1
\end{array}\right), \boldsymbol{X}_{\left(g_{4}\right)}=\left(\begin{array}{cc}
1 & 0 \\
1 & 0 \\
1 & 0 \\
1 & 0 \\
1 & 0 \\
1 & 0 \\
1 & 0 \\
1 & 0 \\
1 & 0 \\
1 & 1 \\
1 & 1 \\
1 & 1
\end{array}\right), \boldsymbol{X}_{\left(g_{5}\right)}=\left(\begin{array}{ccc}
1 & 0 & 0 \\
1 & 0 & 0 \\
1 & 0 & 0 \\
1 & 1 & 0 \\
1 & 1 & 0 \\
1 & 1 & 0 \\
1 & 1 & 0 \\
1 & 1 & 0 \\
1 & 1 & 0 \\
1 & 1 & 1 \\
1 & 1 & 1 \\
1 & 1 & 1
\end{array}\right),
\end{aligned}
$$




$$
\boldsymbol{X}_{\left(g_{6}\right)}=\left(\begin{array}{ccc}
1 & 0 & 0 \\
1 & 0 & 0 \\
1 & 0 & 0 \\
1 & 0 & 0 \\
1 & 0 & 0 \\
1 & 0 & 0 \\
1 & 1 & 0 \\
1 & 1 & 0 \\
1 & 1 & 0 \\
1 & 1 & 1 \\
1 & 1 & 1 \\
1 & 1 & 1
\end{array}\right), \quad \boldsymbol{X}_{\left(g_{7}\right)}=\left(\begin{array}{cccc}
1 & 0 & 0 & 0 \\
1 & 0 & 0 & 0 \\
1 & 0 & 0 & 0 \\
1 & 1 & 0 & 0 \\
1 & 1 & 0 & 0 \\
1 & 1 & 0 & 0 \\
1 & 1 & 1 & 0 \\
1 & 1 & 1 & 0 \\
1 & 1 & 1 & 0 \\
1 & 1 & 1 & 1 \\
1 & 1 & 1 & 1 \\
1 & 1 & 1 & 1
\end{array}\right)
$$

The mean gene expression for each model $g_{r}$ is given by

$$
E\left(Y_{i j} \mid g_{r}\right)=\boldsymbol{X}_{g_{r}} \boldsymbol{\beta}_{r}^{\prime}, \quad r=1, \ldots, R,
$$

where $\boldsymbol{\beta}_{r}$ is the parameter vector for each model given by

$$
\boldsymbol{\beta}_{r}^{\prime}= \begin{cases}\mu_{0}, & \text { model } g_{0}, \\ \left(\mu_{0}, \delta_{1}\right)^{\prime}, & \text { model } g_{1}, \\ \left(\mu_{0}, \delta_{2}\right)^{\prime}, & \text { model } g_{2}, \\ \left(\mu_{0}, \delta_{1}, \delta_{2}\right)^{\prime}, & \text { model } g_{3}, \\ \left(\mu_{0}, \delta_{3}\right)^{\prime}, & \text { model } g_{4}, \\ \left(\mu_{0}, \delta_{1}, \delta_{3}\right)^{\prime}, & \text { model } g_{5}, \\ \left(\mu_{0}, \delta_{2}, \delta_{3}\right)^{\prime}, & \text { model } g_{6}, \\ \left(\mu_{0}, \delta_{1}, \delta_{2}, \delta_{3}\right)^{\prime} & \text { model } g_{7}\end{cases}
$$

\section{MULTIPLICITY ADJUSTMENT}

\subsection{The HESCA Study}

The HESCA dataset (Bijnens et al. 2012) describes results of a dose-response microarray experiment. Human epidermal squamous carcinoma cell line A431 (HESCA431) was grown and cells were stimulated with the growth factor EGF at four concentrations (including placebo) for 24 hours. The gene expression level was measured using GeneChip (Affymetrix). The dataset contains 12 arrays, three arrays for each of four dose levels with 16,998 genes. For details about methodology and preprocessing including normalization, see Bijnens et al. (2012). The aim of the analysis of the HESCA dataset is to detect genes with monotone expression profiles. 


\subsection{The Direct Posterior Probability Approach for Multiplicity Adjustment.}

Due to a high dimensionality of microarray data, the dose-response microarray analysis of the HESCA study requires multiplicity adjustment both within and between genes. Typically, the family wise error rate (FWER) that represents the overall Type I error, i.e., the probability of at least one false rejection of the null hypothesis, and the false discovery rate (FDR), i.e., the expected proportion of the false rejections among all the rejections, are used for the multiplicity adjustment. Following Lin et al. (2012) we apply the FWER method for the multiplicity adjustment within the genes and the FDR for the multiplicity adjustment between the genes. In the following section, we discuss the use of the posterior probability of the null model for the FDR adjustment within the BVS framework.

Assume that there are $m=1, \ldots, M$ genes to analyze simultaneously and the aim is to identify genes that exhibit a monotone relationship with increasing doses of a therapeutic compound. The problem is equivalent to investigating if expression levels of each gene show substantive evidence against the null model $g_{0}$. The posterior probability of the null model $P_{m}\left(g_{0} \mid\right.$ data $)$ holds dual properties as the likelihood of the null model and simultaneously the probability of the false rejection of the null hypothesis when the gene is considered to have the monotone profile (Newton et al. 2007). For a pre-specified threshold $\alpha, P_{m}\left(g_{0} \mid\right.$ data) represents probability of the false positive for the gene $m$. Let $I_{m}$ be an indicator variable of $P_{m}\left(g_{0} \mid\right.$ data $) \leq \alpha$ (i.e., indicator of including gene among genes with "significant" doseresponse relationship). The expected number of the false discoveries (cFD) is

$$
\operatorname{cFD}(\alpha):=\mathrm{E}(\mathrm{cFD})=\sum_{m=1}^{M} P_{m}\left(g_{0} \mid \text { data }\right) I_{m}
$$

Newton et al. (2007) define the conditional (on the data) false discovery rate as

$$
\operatorname{cFDR}(\alpha)=\frac{\mathrm{cFD}(\alpha)}{N(\alpha)}
$$

where $N(\alpha)$ is the number of genes declared significant for a given threshold $\alpha$. Then, the $\operatorname{cFDR}(\alpha)$ represents a mean error made by considering any gene as significant using the threshold $\alpha$. Hence, we select a value of $\alpha$ in a way to keep the $\operatorname{cFDR}(\alpha)$ under the prespecified threshold $\tau$.

Figure 9 shows the relationship between the false discovery rate, the number of significant genes and the threshold for the HESCA case study. As expected, the higher the threshold, the higher the cFDR and the number of significant genes. The implication of this relationship is that in order to control for a certain level of the cFDR, the corresponding threshold can be used as significance level for the posterior probability under the null model. Note that the concept of the significance level as used here is data dependent and consequently, the cFDR control is conditional on the data.

Table 10 shows the number of genes with the significant dose-relationship under the upward and downward monotone profiles from the HESCA study. The number of genes with the 
Table 10: Number of rejected null hypotheses according to the frequentist methods and to the BVS model while controlling the FDR and the cFDR on a 0.05 level, respectively.

\begin{tabular}{lrrrr}
\hline \hline Profile & LRT & MCT(W) & MCT(M) & BVS \\
\hline Upward & 2057 & 1772 & 1954 & 1634 \\
Downward & 2464 & 2053 & 2364 & 1798 \\
\hline
\end{tabular}

significant dose-response relationship is higher for the frequentist methods than the BVS model at $5 \%$ false discovery rate. At a fixed level of FDR, the higher number of the significant genes for the frequentist methods may imply the better power with these methods than with the BVS model since the power is often associated with the number of the significant genes. However, the FDR controlled by the frequentist methods and the cFDR introduced for the BVS context are not entirely same quantities, since one arise from an adjustment of p-values and other one from an adjustment of the posterior probabilities of the null model. The comparison of the BVS model and the frequentists methods in terms of the Type I error and the power is investigated through a simulation study presented in the next section.
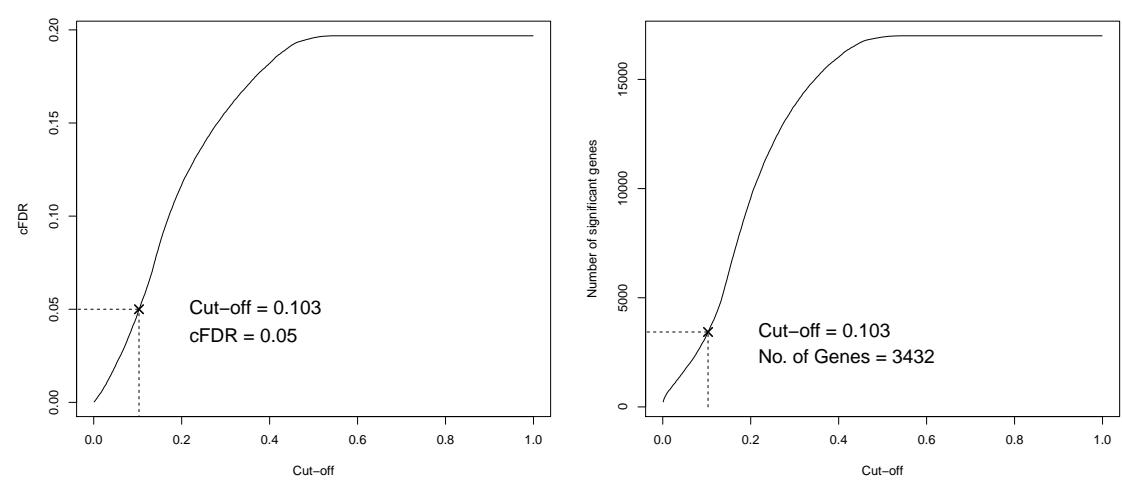

Figure 9: Adjustment for the multiplicity for the HESCA data. Left panel: the relationship between the conditional false discovery rate (cFDR) and the cut-off values. Right panel: the relationship between the number of significant genes and the cut-off values.

\section{References}

Barlow, R. E., Bartholomew, D. J., Bremner, M. J., and Brunk, H. D. (1972), Statistical Inference under Order Restriction, New York: John Wiley \& Sons.

Bijnens, L., Göhlmann, H. W., Lin, D., Talloen, W., Perera, T., Van Den Wyngaert, I., De Ridder, F., De Bondt, A., and Peeters, P. (2012), Modeling Dose-response Microarray Data 
Table 11: The set of 16 possible monotonic dose-response models for an experiment with five dose levels (including placebo). Denote $\mu_{i}$ the mean response of the dose level. The model $g_{0}$ represents the null model of no dose effect.

\begin{tabular}{cll}
\hline \hline Model & Up: Mean Structure & Down: Mean Structure \\
\hline$g_{0}$ & $\mu_{0}=\mu_{1}=\mu_{2}=\mu_{3}=\mu_{4}$ & $\mu_{0}=\mu_{1}=\mu_{2}=\mu_{3}=\mu_{4}$ \\
$g_{1}$ & $\mu_{0}<\mu_{1}=\mu_{2}=\mu_{3}=\mu_{4}$ & $\mu_{0}>\mu_{1}=\mu_{2}=\mu_{3}=\mu_{4}$ \\
$g_{2}$ & $\mu_{0}=\mu_{1}<\mu_{2}=\mu_{3}=\mu_{4}$ & $\mu_{0}=\mu_{1}>\mu_{2}=\mu_{3}=\mu_{4}$ \\
$g_{3}$ & $\mu_{0}<\mu_{1}<\mu_{2}=\mu_{3}=\mu_{4}$ & $\mu_{0}>\mu_{1}>\mu_{2}=\mu_{3}=\mu_{4}$ \\
$g_{4}$ & $\mu_{0}=\mu_{1}=\mu_{2}<\mu_{3}=\mu_{4}$ & $\mu_{0}=\mu_{1}=\mu_{2}>\mu_{3}=\mu_{4}$ \\
$g_{5}$ & $\mu_{0}<\mu_{1}=\mu_{2}<\mu_{3}=\mu_{4}$ & $\mu_{0}>\mu_{1}=\mu_{2}>\mu_{3}=\mu_{4}$ \\
$g_{6}$ & $\mu_{0}=\mu_{1}<\mu_{2}<\mu_{3}=\mu_{4}$ & $\mu_{0}=\mu_{1}>\mu_{2}>\mu_{3}=\mu_{4}$ \\
$g_{7}$ & $\mu_{0}<\mu_{1}<\mu_{2}<\mu_{3}=\mu_{4}$ & $\mu_{0}>\mu_{1}>\mu_{2}>\mu_{3}=\mu_{4}$ \\
$g_{8}$ & $\mu_{0}=\mu_{1}=\mu_{2}=\mu_{3}<\mu_{4}$ & $\mu_{0}=\mu_{1}=\mu_{2}=\mu_{3}>\mu_{4}$ \\
$g_{9}$ & $\mu_{0}<\mu_{1}=\mu_{2}=\mu_{3}<\mu_{4}$ & $\mu_{0}>\mu_{1}=\mu_{2}=\mu_{3}>\mu_{4}$ \\
$g_{10}$ & $\mu_{0}=\mu_{1}<\mu_{2}=\mu_{3}<\mu_{4}$ & $\mu_{0}=\mu_{1}>\mu_{2}=\mu_{3}>\mu_{4}$ \\
$g_{11}$ & $\mu_{0}<\mu_{1}<\mu_{2}=\mu_{3}<\mu_{4}$ & $\mu_{0}>\mu_{1}>\mu_{2}=\mu_{3}>\mu_{4}$ \\
$g_{12}$ & $\mu_{0}=\mu_{1}=\mu_{2}<\mu_{3}<\mu_{4}$ & $\mu_{0}=\mu_{1}=\mu_{2}>\mu_{3}>\mu_{4}$ \\
$g_{13}$ & $\mu_{0}<\mu_{1}=\mu_{2}<\mu_{3}<\mu_{4}$ & $\mu_{0}>\mu_{1}=\mu_{2}>\mu_{3}>\mu_{4}$ \\
$g_{14}$ & $\mu_{0}=\mu_{1}<\mu_{2}<\mu_{3}<\mu_{4}$ & $\mu_{0}=\mu_{1}>\mu_{2}>\mu_{3}>\mu_{4}$ \\
$g_{15}$ & $\mu_{0}<\mu_{1}<\mu_{2}<\mu_{3}<\mu_{4}$ & $\mu_{0}>\mu_{1}>\mu_{2}>\mu_{3}>\mu_{4}$ \\
\hline
\end{tabular}

in Early Drug Development Experiments Using R, Springer, chap. Functional Genomics Dose-Response Experiments, pp. 69-80.

Bretz, F. (1999), "Powerful Modification on Williams' Test on Trend," Ph.D. thesis, Universität Hannover.

Hothorn, L. A. (2006), "Multiple comparisons and multiple contrasts in randomized doseresponse trials-confidence interval oriented approaches." Journal of Biopharmaceutical Statistics, 16(5), 711-731.

Lin, D., Hothorn, L. A., Djira, G. D., and Bretz, F. (2012), Modeling Dose-response Microarray Data in Early Drug Development Experiments Using $R$, Springer, chap. Multiple Contrast Tests for Testing Dose-response Relationships Under Order Restricted Alternatives, pp. 233-247.

Marcus, R. (1976), "The Powers of Some Tests of the Equality of Normal Means against an Ordered Alternative," Biometrika, 63, 177-183.

Newton, M., Wang, P., and Kendziorski, C. (2007), Bayesian Inference for gene expres- 
Table 12: Setting matrix for $K=4$ before multiplying by $\lambda=1,1.5,2,2.5,3$.

\begin{tabular}{rrrrr}
\hline \hline Profile & Dose 0 & Dose 1 & Dose 2 & Dose 3 \\
\hline$g_{1}$ & 1.15 & 2.31 & 2.31 & 2.31 \\
$g_{2}$ & 1.00 & 1.00 & 2.00 & 2.00 \\
$g_{3}$ & 0.60 & 1.21 & 1.81 & 1.81 \\
$g_{4}$ & 1.15 & 1.15 & 1.15 & 2.31 \\
$g_{5}$ & 0.71 & 1.41 & 1.41 & 2.12 \\
$g_{6}$ & 0.60 & 0.60 & 1.21 & 1.81 \\
$g_{7}$ & 0.45 & 0.89 & 1.34 & 1.79 \\
\hline
\end{tabular}

sion and proteomics, Cambridge university press, chap. Hierarchical mixture models for expression profiles, pp. 40-52.

Robertson, T., Wright, F. T., and Dykstra, R. L. (1988), Order Restricted Statistical Inference., John Wiley \& Sons Ltd.

Williams, D. A. (1971), "A Test for Differences between Treatment Means When Several Dose Levels Are Compared with a Zero Dose Control," Biometrics, 27, 103-117. 
Table 13: Setting matrix for $K=5$ before multiplying by $\lambda=1,1.5,2,2.5,3$.

\begin{tabular}{rrrrrr}
\hline \hline Profile & Dose 0 & Dose 1 & Dose 2 & Dose 3 & Dose 4 \\
\hline$g_{1}$ & 1.12 & 2.24 & 2.24 & 2.24 & 2.24 \\
$g_{2}$ & 0.91 & 0.91 & 1.83 & 1.83 & 1.83 \\
$g_{3}$ & 0.56 & 1.12 & 1.68 & 1.68 & 1.68 \\
$g_{4}$ & 0.91 & 0.91 & 0.91 & 1.83 & 1.83 \\
$g_{5}$ & 0.60 & 1.20 & 1.20 & 1.79 & 1.79 \\
$g_{6}$ & 0.56 & 0.56 & 1.12 & 1.68 & 1.68 \\
$g_{7}$ & 0.40 & 0.79 & 1.19 & 1.58 & 1.58 \\
$g_{8}$ & 1.12 & 1.12 & 1.12 & 1.12 & 2.24 \\
$g_{9}$ & 0.71 & 1.41 & 1.41 & 1.41 & 2.12 \\
$g_{1} 0$ & 0.60 & 0.60 & 1.20 & 1.20 & 1.79 \\
$g_{1} 1$ & 0.44 & 0.88 & 1.32 & 1.32 & 1.75 \\
$g_{1} 2$ & 0.56 & 0.56 & 0.56 & 1.12 & 1.68 \\
$g_{1} 3$ & 0.44 & 0.88 & 0.88 & 1.32 & 1.75 \\
$g_{1} 4$ & 0.40 & 0.40 & 0.79 & 1.19 & 1.58 \\
$g_{1} 5$ & 0.32 & 0.63 & 0.95 & 1.26 & 1.58 \\
\hline
\end{tabular}

